\title{
Bounds and prospects for stable multiply charged particles at the LHC
}

\author{
Sebastian Jäger, ${ }^{a}$ Sandra Kvedaraitè, ${ }^{a}$ Gilad Perez $^{b}$ and Inbar Savoray ${ }^{b}$ \\ ${ }^{a}$ Department of Physics and Astronomy, University of Sussex, \\ Brighton, BN1 9QH, U.K. \\ ${ }^{b}$ Department of Particle Physics and Astrophysics, Weizmann Institute of Science, \\ Rehovot 7610001, Israel
}

E-mail: S.Jaeger@sussex.ac.uk, S.Kvedaraite@sussex.ac.uk, gilad.perez@weizmann.ac.il, inbar.savoray@weizmann.ac.il

ABSTRACT: Colored and colorless particles that are stable on collider scales and carry exotic electric charges, so-called Multiply-Charged Heavy Stable Particles (MCHSPs), exist in extensions of the Standard Model, and can include the top partner(s) in solutions of the hierarchy problem. To obtain bounds on color-triplets and color-singlets of charges up to $|Q|=8$, we recast searches for signatures of two production channels: the "open" channel - where the particles are pair-produced above threshold, and are detectable in dedicated LHC searches for stable multiply charged leptons, and the "closed" channel where a particle-antiparticle pair is produced as a bound state, detectable in searches for a diphoton resonance. We recast the open lepton searches by incorporating the relevant strong-interaction effects for color-triplets. In both open and closed production, we provide a careful assessment of photon-induced processes using the accurate LUXqed PDF, resulting in substantially weaker bounds than previously claimed in the literature for the colorless case. Our bounds for colored MCHSPs are shown for the first time, as the LHC experiments have not searched for them directly. Generally, we obtain nearly charge-independent lower mass limits of around $970 \mathrm{GeV}$ (color-triplet scalar), $1200 \mathrm{GeV}$ (color-triplet fermion), and 880-900 GeV (color-singlet fermion) from open production, and strongly charge-dependent limits from closed production. In all cases there is a cross-over between dominance by open and closed searches at some charge. We provide prospective bounds for $\sqrt{s}=13 \mathrm{TeV}$ LHC searches at integrated luminosities of $39.5 \mathrm{fb}^{-1}, 100 \mathrm{fb}^{-1}$, and $300 \mathrm{fb}^{-1}$. Moreover, we show that a joint observation in the open and the closed channels allows to determine the mass, spin, color, and electric charge of the particle.

KeYwords: Phenomenological Models, Supersymmetry Phenomenology

ARXIV EPRINT: 1812.03182 


\section{Contents}

1 Introduction 1

2 Stable multiply-charged particles at the LHC 3

2.1 Recalculating production cross sections 5

2.2 Efficiency calculation 5

2.2.1 Procedure 6

2.2.2 Validation 9

3 Bound state signal at the LHC $\quad 10$

4 Current status - recast bounds $\quad 13$

$\begin{array}{lll}\text { 4.1 Bounds from open signatures of MCHSPs } & 14\end{array}$

4.2 Bounds from closed signatures of Multiply-Charged Heavy Stable Particles $\begin{array}{lr}\text { (MCHSPs) } & 16\end{array}$

$\begin{array}{lll}4.3 & \text { Combined bounds } & 16\end{array}$

$\begin{array}{ll}\text { 4.4 The leptonic case - comparison to the literature } & 17\end{array}$

5 Future scenarios — discovery and exclusion $\quad 18$

6 Conclusions and outlook 20

$\begin{array}{ll}\text { A Open-production signatures } & 23\end{array}$

A.1 Cross sections 23

$\begin{array}{lll}\text { A.2 Simplified efficiency calculation } & 25\end{array}$

$\begin{array}{ll}\text { A.2.1 Time of Flight (TOF) calculation } & 25\end{array}$

$\begin{array}{lll}\text { A.2.2 Straight tracks approximation } & 25\end{array}$

$\begin{array}{ll}\text { A.2.3 Global muon offline selection } & 26\end{array}$

$\begin{array}{lll}\text { A.2.4 Efficiency values } & 27\end{array}$

$\begin{array}{lll}\text { A.3 } & \text { Effective cross sections } & 31\end{array}$

B Closed-production signatures - diphoton cross sections 34

\section{Introduction}

Extensions of the Standard Model (SM) often contain particles that are stable, or sufficiently long-lived to be effectively stable on the time and distance scales relevant to collider experiments. Examples include the lightest supersymmetric particle if $R$-parity is approximately or exactly conserved (see [1] for a review) and particles in certain composite Higgs 


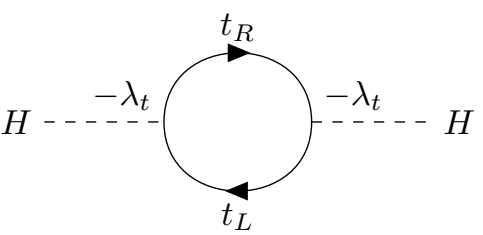

(a)

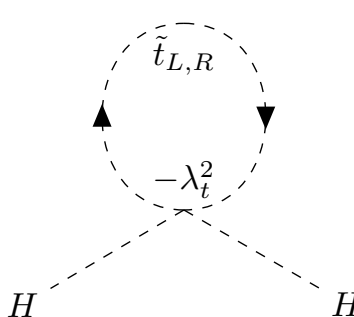

(b)

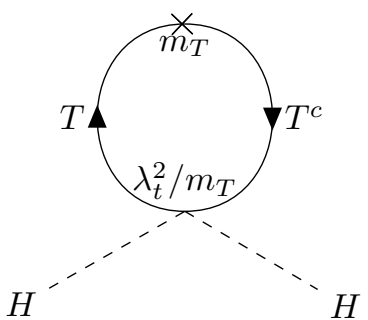

(c)

Figure 1. (a) Divergent top loop correction to the Higgs mass. (b) Loop contribution of a scalar top-partner. (c) Loop contribution of a fermion top-partner. The diagrams are taken from [4].

models [2]. It is possible that such a particle has exotic and possibly large electric charge; we will refer to this as a MCHSP.

Within the context of the naturalness problem (see e.g [3]), such MCHSP can cure the quadratic divergence in the Higgs mass parameter; this has recently been realized in the framework of Colorful Twisted Top Partners (CTTPs) [4]. The CTTP can take the form of a spin- 0 or spin- $1 / 2$ color-triplet of arbitrary electric charge. The divergence cancellation occurs between the top loop in figure 1(a), and a scalar CTTP loop (figure 1(b)) or a fermion loop (figure 1(c)). The CTTP is stable either due to an (approximate) accidental $\mathrm{U}(1)$ symmetry, conserving partner-number, or due to an (approximate) $Z_{2}$ symmetry, under which the CTTP is odd and all SM particles are even. In fact, CTTPs of charges different from $Q=2 / 3+n$ or $Q=-(1 / 3+n)$, where $\mathrm{n}$ is a non-negative integer, are not allowed to decay to SM particles altogether [5]. Consequently, exotically charged top partners are likely to be stable or long lived.

Motivated by the above, we will consider color-triplet particles with arbitrary electric charges, and refer to them as CTTPs, or "partners", irrespective of whether they are connected to naturalness or not. An important implication of their long lifetime is the presence of a near-threshold, positronium-like bound state. In the top partner case, this is known as the partnerium [4], and we will use this term to denote the bound state in the generic case. The colored particle-antiparticle pair is bound by both a Coulomblike Quantum Chromodynamics (QCD) potential and by Electromagnetism (EM), with the latter becoming important for large charges. Since partnerium carries no conserved charge, it is free to annihilate into SM particles, leaving potentially detectable signatures, the most relevant of which, for our purposes, is a diphoton resonance.

In addition to the bound-state production (referred to as "closed"), the stable (or long-lived) partner can be pair-produced above threshold (referred to as "open"), leaving tracks in all detector layers and eventually escaping without an observed decay. Colortriplet top partners with charges different than $2 / 3$ have not been directly searched for at the LHC, and are largely unconstrained. In this work, we obtain current bounds on exotically-charged scalar and fermion CTTPs, considering both open pair production and partnerium signatures. We also obtain prospective bounds, for future LHC searches, at several integrated luminosities and Center of Mass (COM) energy of $13 \mathrm{TeV}$. We choose 
to focus on multiply-charged $(|Q|>1)$ color-triplet top partners, which are expected to exhibit an interesting interplay between the two channels, especially given their sizable partnerium-annihilation to a pair of photons. In addition, we consider color-singlet fermion MCHSPs, referred to as lepton-like particles. In this case, the bound state is purely EM, referred to as "leptonium". We restrict ourselves to $\mathrm{SU}(2)_{\text {weak }}$ singlets, both for colored and colorless MCHSPs.

The remainder of the paper is organized as follows. In section 2 we discuss the openproduction signatures of MCHSPs, and consider the existing run-I $(\sqrt{s}=8 \mathrm{TeV}) \mathrm{LHC}$ searches for color-neutral stable particles with large electric charges. In order to recast these searches for colored particles, and to update their results for colorless particles, we compute the production cross sections and the detection efficiencies for both spinless and spin- $1 / 2$ color-triplets, and for colorless fermions, all with charges $Q$ in the range $1 \leq|Q| \leq 8$ and masses $m$ in the range $100 \mathrm{GeV} \leq m \leq 3 \mathrm{TeV}$. We validate our methodology against the published efficiencies in the colorless case. We also obtain the required components for the prospective $\sqrt{s}=13 \mathrm{TeV}$ searches. Section 3 reviews the pertinent aspects of the bound state signatures, in particular the resonant-production cross section of a diphoton final state. Section 4 contains our main findings, in the form of current lower limits on the masses of colored and color-neutral particles. For the color-neutral case, we obtain weaker constraints than a recent paper, albeit stronger than the bounds originally obtained by CMS; we trace these discrepancies to the photon-induced component of the signal and stress the importance of an appropriate choice of the photon Parton Distribution Function (PDF). In section 5, we present projected bounds for LHC searches at $\sqrt{s}=13 \mathrm{TeV}$, for integrated luminosities of $39.5 \mathrm{fb}^{-1}, 100 \mathrm{fb}^{-1}$, and $300 \mathrm{fb}^{-1}$, taking into account the scaling of pileup. We briefly discuss how by combining an open-production effective cross section measurement and a diphoton resonance observation one can determine the mass, spin, electric charge and color charge of the particle. Our conclusions can be found in section 6 .

\section{Stable multiply-charged particles at the LHC}

Our first goal is to obtain constraints on CTTPs from their signatures as stable particles, produced above threshold. So far, there have been no LHC searches designated for colortriplet MCHSPs. However, there have been experimental searches for other kinds of heavy stable charged particles, which could be potentially recast to apply to CTTPs.

The stable fermion and scalar color-triplet partners are expected to hadronize to form "R-hadrons", similarly to quarks and squarks [6]. Searches for stable R-hadrons have been carried out both in ATLAS [7-9], and in CMS [10-12] for COM energies of 7, 8 and $13 \mathrm{TeV}$. However, these searches are designated for stops and gluinos, and thus optimized for unitcharged R-hadrons. Applying such searches for multiply-charged R-hadrons could bear a significant loss of the discovery potential.

Searches for multiply-charged color-singlet fermions account for the difficulties concerning the detection of MCHSPs. These searches were conducted by ATLAS for particles with charges of 2-6 [13], and conducted by CMS for particles with charges of 1-8 [11]. Both searches were analyzed for $\sqrt{s}=8 \mathrm{TeV}$, but have yet to be updated for $\sqrt{s}=13 \mathrm{TeV}$. 
Results for a $Q=2$ lepton-like particle have been published by CMS for $\sqrt{s}=13 \mathrm{TeV}$, following an analysis that uses the same discriminators as for R-hadrons [10]. However, the resulting bound was less stringent than the one derived from the designated search for multiply-charged lepton-like particles, carried out for $\sqrt{s}=8 \mathrm{TeV}$.

As the aforementioned searches were carried out for colorless fermions only, heavy stable CTTPs are still essentially unconstrained. While multiply-charged scalar and fermion CTTPs are expected to share a lot of phenomenological traits with multiply-charged leptons, QCD-induced processes for color-triplets still need to be accounted for. First, one should consider the appropriate production mechanism, both for cross section and for efficiency calculations. Second, the hadronization of the colored particle-pair might yield two differently charged R-hadrons, and thus change the event acceptance. Moreover, nuclear energy loss and charge-changing effects [6] might further reduce the efficiency of the search. Therefore, the existing analyses are not sufficient for obtaining bounds on stable CTTPs.

Furthermore, the previous analyses for colorless fermions might be lacking. As shown in the re-analyses of the ATLAS search [13] in [14], the bounds on multiply-charged particles are sensitive to the treatment of photo-induced processes, which were not included in the original LHC analyses. However, the PDF used in [14] has been shown to have large uncertainties for the photon PDF and thus also for the photon luminosity [15, 16, 40]. This translates into large uncertainties on the previously obtained bounds. A more accurate determination of the photon PDF using ep scattering data was proposed in refs. [15, 18], resulting in significantly smaller errors, which are at the $1 \%$ level over a large range of momentum fractions. For these reasons, we would like to reanalyze the signatures of MCHSPs using the resulting LUXqed PDF [15].

This motivates us to recast a search for lepton-like MCHSPs, in order to apply its observations to fermion and scalar CTTPs, and to update the bounds on lepton-like particles. The rest of this section is dedicated to describing our recast procedure.

We chose to recast the most recent CMS search for lepton-like particles with charges of 1-8 [11]. ${ }^{1}$ Since the search is a counting experiment, essentially blind to mass and charge, it is imposing a universal upper limit on the product of the cross section and the efficiency, $\sigma \cdot \epsilon$. This "effective cross section" upper limit is then compared to its theoretical prediction for each signal benchmark, described below, to obtain the upper bounds on the signal mass. In the next sections, we discuss our calculations of the cross sections and efficiencies separately, which are later combined to obtain the theoretical effective cross sections. As the search is only available for $\sqrt{s}=7 \& 8 \mathrm{TeV}$, we calculate the bounds based on the observed result at $\sqrt{s}=8 \mathrm{TeV}$, and estimate the expected bounds for $\sqrt{s}=13 \mathrm{TeV}$.

For convenience, our signal benchmarks are based on the charges already considered in the original search. Namely, color-singlets with integer charges $\left|Q_{\mathrm{LLP}}\right|=1-8$ and color-triplets that hadronize to acquire such charges, initially charged as: $5 / 3 \leq Q_{\mathrm{CTTP}} \leq$ $23 / 3$ and $-22 / 3 \leq Q_{\mathrm{CTTP}} \leq-4 / 3$, in increments of one. We did not include charges of $-1 / 3$ and $2 / 3$ in our analysis, as those were better studied in stable R-hadrons searches.

\footnotetext{
${ }^{1}$ The corresponding ATLAS search [13] resulted in similar bounds, and should have the same qualitative efficiency behavior, however it was only applied to $Q \leq 6$.
} 
Charges of $26 / 3$ and $-25 / 3$ were disregarded due to their sizable hadronization fraction to $\left|Q_{\mathrm{R} \text {-hadron }}\right|=9$ particles, that were not included in the original search. It has been shown in $[14,17]$ that particles with such large charges can still be treated perturbatively as long as the coupling is sufficiently small and the energy domain is well below the Landau pole. This is ensured when $\alpha Q^{2} \lesssim \mathcal{O}(1)$. As the theory loses perturbativity for $\alpha Q^{2} \gtrsim \mathcal{O}(1)$, our predictions could not be straightforwardly extrapolated for $Q \gtrsim 10$. Since both the observations and the selections of the search are common to all masses and charges, one can easily interpolate our results for any intermediate charge.

The masses of the signal benchmarks were determined in a similar fashion. Since the original search considered masses of $100-1000 \mathrm{GeV}$, lepton-like particles of the same masses were generated in a Monte-Carlo simulation, described in the following, in order to estimate the accuracy of the efficiency calculation. Bounds were calculated for particles of masses $500-3000 \mathrm{GeV}$.

\subsection{Recalculating production cross sections}

The pair-production cross section of CTTPs is calculated by summing the contributions from the $g g, g \gamma$ and $\gamma \gamma$ Vector Boson Fusion (VBF) production channels, as well as from the $q \bar{q}$ Drell-Yan (DY) production channel, mediated by $g, \gamma$ or $Z$. The calculation of the pairproduction cross section of lepton-like particles accounts for production both by photonfusion and by a DY process mediated by $\gamma$ or $Z$. In contrast to both the original search [11] and to a re-interpretation of the ATLAS search [13] in [14], all cross sections below are calculated with the LUXqed PDF set (LUXqed17_plus_PDF4LHC15_nnlo_100) [15, 18]. We use MadGraph5 [19] to calculate the parton-level cross section at LO. The resulting cross sections are presented in appendix A.

The relative importance of the different production channels is highly affected by the PDF of the incoming partons. Photon-induced charge-dependent VBF processes are suppressed by the smallness of the photon PDF, while charge-independent gluon-fusion processes benefit from the large PDF of the gluon. Since the ratio between the gluon PDF and the photon PDF is slightly smaller at higher energies, a large charge-dependent contribution could eventually overcome the PDFs imbalance. Thus, as shown in figure 2, heavier particles with large charges will mostly be produced by photon-inclusive, highly chargedependent processes, and lighter particles with small charges will mostly be produced by charge-independent processes.

We use Pythia8 $[20,21]$ to perform showering and hadronization. As can be seen in table 1 , hadronized partners mainly have charges of $\pm(Q+1 / 3)$ and $\pm(Q-2 / 3)$, with only a negligible fraction of $\pm(Q+4 / 3)$ R-hadrons. Since hadronization of the heavy partner and anti-partner takes place mostly independently, they may hadronize into two differently charged R-hadrons.

\subsection{Efficiency calculation}

Since we do not have access to the full CMS detector simulation, we defined a set of selection criteria to account for detection efficiencies. Using our efficiency calculation, with the production mechanism described in [11], we aim to reproduce the mass bounds 


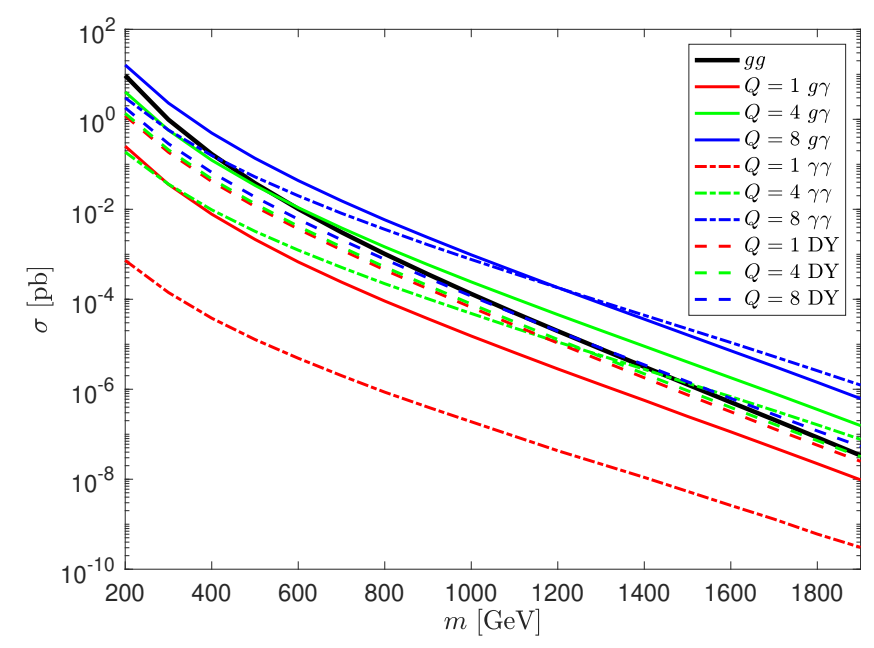

Figure 2. Different subprocesses for pair-production of a scalar CTTP with charges of $Q=1,4,8$.

\begin{tabular}{|l|r|}
\hline R-hadron & Fraction $(\%)$ \\
\hline$R^{Q+1 / 3}$ & 28.25 \\
$R^{Q-2 / 3}$ & 21.50 \\
$R^{Q+4 / 3}$ & 0.25 \\
$\bar{R}^{-(Q+1 / 3)}$ & 26.75 \\
$\bar{R}^{-(Q-2 / 3)}$ & 23.00 \\
$\bar{R}^{-(Q+4 / 3)}$ & 0.25 \\
\hline
\end{tabular}

Table 1. Fractions of produced R-hadrons with specific charges, obtained using MadGraph and Pythia simulation of partner pair-production and hadronization.

obtained by CMS for lepton-like particles within 15\% accuracy. A similar accuracy should be maintained as we calculate the bounds on the masses of CTTPs, and of lepton-like particles produced as in section 2.1. We account for the online, offline and final selections criteria, as will be explained in the following paragraphs. Even though our treatment is somewhat rough, we will see it is more than satisfactory for obtaining mass bounds, as they are only weakly affected by efficiencies.

\subsubsection{Procedure}

The online selection for the search [11] consists of an $E_{T}^{\text {miss }}$ trigger and/or a muon trigger. To pass the $E_{T}^{\text {miss }}$ trigger, an event should be assigned $E_{T}^{\text {miss }} \geq 150 \mathrm{GeV}$ as measured in the calorimeter. This criterion is useful to some extent for particles that were not reconstructed as muons, but we expect it to have a negligible contribution to the overall efficiency, since the offline and final selections essentially require a muon candidate.

We therefore focus on simulating the muon trigger as our online selection. To pass the muon trigger requirements, an event must have at least one particle reconstructed as a muon. The muon candidate must have $\eta \leq 2.1$, and $p_{T_{\text {meas }}} \geq 40 \mathrm{GeV}$ as measured in the 
Inner Detector (ID). The transverse momentum is measured from the curvature radius of the particle's track, $r$, under a magnetic field, $B$, which follows

$$
r=\frac{p_{T}}{0.3 \cdot Q \cdot B}
$$

However, the reconstruction algorithm assumes $Q=1$, and so the measured $p_{T}$ is $p_{T_{\text {meas }}}=$ $p_{T_{\text {truth }}} / Q$. This effectively requires the truth-level transverse momentum to satisfy $p_{T_{\text {truth }}} \geq$ $Q \cdot(40 \mathrm{GeV})$, thus reducing the efficiency for large charges and small masses.

In addition, triggering particles must be fast enough to have both their ID and Muon System (MS) tracks in the same bunch crossing [22]. Since the LHC collisions were planned to occur every $25 \mathrm{~ns}$, slow particles that reach the MS more than $25 \mathrm{~ns}$ after a $\beta=1$ particle, will be associated with the wrong bunch crossing and thus will not have a matching ID track [23]. An additional Resistive Plate Chamber (RPC) muon trigger was applied for $\eta \leq 1.6$, allowing candidates to reach the MS up to $50 \mathrm{~ns}$ later than a $\beta=1$ particle [24].

RPC-triggered particles must have a minimum of four RPC hits (three if not geometrically possible) within the trigger time window [24, 25]. A similar requirement also holds for particles triggered by the Cathode Strip Chambers (CSCs) positioned at $\eta \geq 1.6$, as the CSC trigger relies on three different track segments to reconstruct $p_{T}[26]$. These constraints effectively define a minimal distance, denoted as $x_{\text {trigger }}$, that candidates must travel within the trigger time window, as function of $\eta$.

In order to calculate the time required for a candidate to travel the distance necessary for triggering, denoted as $t_{\mathrm{TOF}}$, one must account for the ionization energy loss in the Hadronic Calorimeter (HCAL) and in the MS. Following the Bethe-Bloch formula [27], the ionization energy loss rate decreases with the velocity of the particle and quadratically increases with its charge. Therefore, the timing requirement is expected to be crucial for MCHSPs, that are both produced with smaller velocities and significantly slowed down, or even stopped, by ionization energy loss.

Heavy R-hadrons may also undergo nuclear interactions with matter, causing additional energy loss and potentially altering the quark content of the R-hadron, resulting in a charge change [6]. However, as can be seen in figure 3, for slow particles with large charges, nuclear energy loss is quite negligible compared to ionization energy loss, and hence could be ignored. Since we did not have access to a reliable simulation of chargechanging processes, we could not account for them in our analysis. As we would expect these processes to cause some efficiency loss, it would be desirable to include them in a full experimental study. The calculation of $t_{\mathrm{TOF}}$ is further explained in appendix. A.2.1.

Candidates in events passing the online selection are subject to an offline selection specified in tables 1-2 of [11], applied at particle level. Our offline efficiency calculation is rather limited, and only explicitly includes $p_{T}$ and isolation criteria, as described in lines 4-5 of table 2. An additional selection requires the particle to be reconstructed as a global muon [30], filtering out particles that were not identified as muons at the muon trigger level. Therefore, we replaced the global muon selection by only accepting candidates that individually satisfy the online muon trigger requirements, as defined above. This assumption is further justified in appendix A.2.3. Since we cannot account for the 


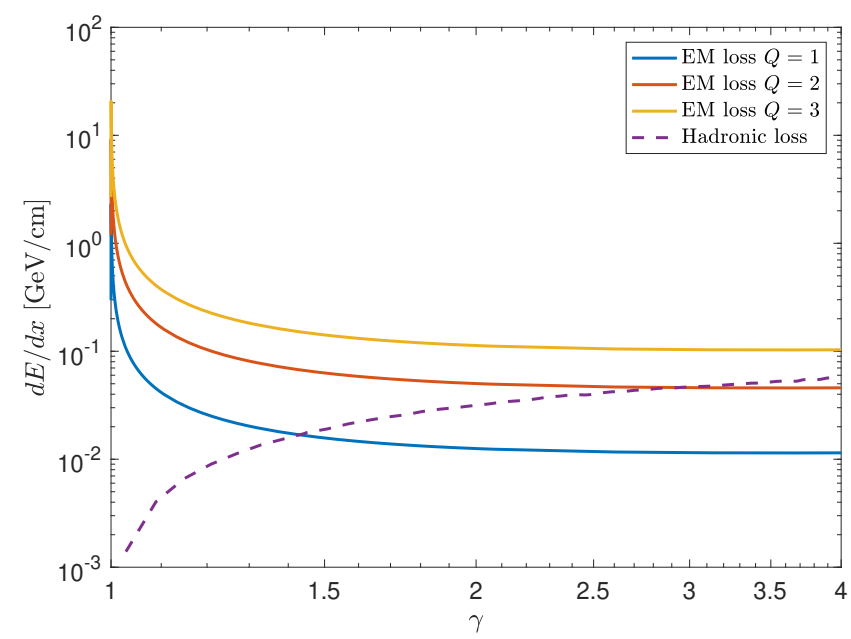

Figure 3. Energy loss per distance traveled in iron as a function of $\gamma$. Solid - ionization energy loss for $Q=1,2,3$ [28]. Dashed - average nuclear energy loss for a hadronized stable stop [29].

remaining criteria without a full detector simulation, we use the values quoted in tables $\mathrm{C} 1-\mathrm{C} 16$ of [23] as multiplicative factors for the offline efficiency calculation. A factor for each signal mass and charge is calculated by

$$
\epsilon_{\mathrm{offline}}^{\text {sim }}=\frac{\epsilon_{\mathrm{offline}}}{\epsilon_{\text {global muon }} \cdot \epsilon_{p_{T}} \cdot \epsilon_{\text {isolation }}},
$$

where $\epsilon_{\text {offline }}$ is the fraction of particles passing the offline selection, out of all particles from events that passed the online selection. The efficiencies $\epsilon_{\text {global muon }}, \epsilon_{p_{T}}, \epsilon_{\text {isolation }}$ correspond to the fractions of particles passing the global muon, $p_{T}$ and $\sum_{R \leq 0.3} p_{T}$ requirements, respectively, out of the particles passing all selections imposed prior to them (online selection included). The aforementioned values were given in [23] for lepton-like particles of charges 1-8 and masses of 100-1000 GeV. Since they vary weakly with mass, we use $m=1000 \mathrm{GeV}$ efficiencies for all $m \geq 1000 \mathrm{GeV}$ particles.

Lastly, the signal region is determined by the final selection criteria, presented in table 3 (line 4) of [11]. We include the $1 / \beta \geq 1.2$ selection in our criteria, designed to identify slow particles, and calculate it using the TOF defined in eq. A.1. Since we cannot recreate the $I_{\text {as }}$ selection, we expect our efficiency to be overestimated for unit-charge particles. However, particles with larger charges are not affected [23].

Our efficiency calculation may require adjustment for $\sqrt{s}=13 \mathrm{TeV}$. In the absence of MCHSPs searches at $\sqrt{s}=13 \mathrm{TeV}$, we have to make certain assumptions about how the selection criteria will change. The choice of $p_{T}$ thresholds is taken from the $\sqrt{s}=$ $13 \mathrm{TeV}$ search for unit-charged heavy stable charged particles [10], since the corresponding $\sqrt{s}=8 \mathrm{TeV}$ searches for multiply-charged and unit-charged particles had the same $p_{T}$ requirements. We had no reliable estimate of how the offline and the final selections might be modified for $13 \mathrm{TeV}$. We therefore kept them the same as in $8 \mathrm{TeV}$ searches, noting that the offline efficiencies given in [23] for the $7 \mathrm{TeV}$ and the $8 \mathrm{TeV}$ runs show only a weak dependence on the masses and COM energies. 


\begin{tabular}{|c|c|c|}
\hline \multirow{2}{*}{ Online } & $\mathbf{8 ~ T e V}$ & $\mathbf{1 3} \mathbf{~ T e V}$ \\
\hline \multirow{2}{*}{ Offline } & $p_{T} \geq Q \cdot 40 \mathrm{GeV}$ & $p_{T} \geq Q \cdot 50 \mathrm{GeV}$ \\
\cline { 2 - 3 } & \multicolumn{2}{|c|}{$|\eta| \leq 2.1$} \\
\cline { 2 - 3 } & $t_{\mathrm{TOF}}-\frac{x_{\text {trigger }}}{c} \leq 50 \mathrm{~ns}(25 \mathrm{~ns})$ \\
\hline \multirow{2}{*}{ Final } & $p_{T} \geq Q \cdot 45 \mathrm{GeV}$ & $p_{T} \geq Q \cdot 55 \mathrm{GeV}$ \\
\cline { 2 - 3 } & \multicolumn{2}{|c|}{$\sum_{R \leq 0.3} p_{T} \leq 50 \mathrm{GeV}$} \\
\hline \multirow{2}{*}{ Multiplicative Factor } & \multicolumn{2}{|c|}{$x_{\text {trigger }} \geq 1.2$} \\
\hline \multirow{2}{*}{$\begin{array}{c}\text { sim } \\
\text { sim }\end{array}$} & $\left.\epsilon_{\text {offline }}^{\text {sim }}(Q, m), m \leq 1000\right), m>1000 \mathrm{GeV}$ \\
\hline
\end{tabular}

Table 2. Simplified efficiency calculation steps and criteria used in this analysis. Each step is applied only to candidates passing the selections in the steps above it. The online timing requirement is $50 \mathrm{~ns}$ for $|\eta| \leq 1.6$ and $25 \mathrm{~ns}$ for $|\eta|>1.6$. The multiplicative factor accounts for the offline selection criteria, which are not explicitly simulated, and instead the efficiencies associated with them are taken from [23]. More details in text.

The efficiency calculation steps and criteria are summarized in table 2. Events that pass those criteria are assumed $100 \%$ efficiency, as our calculation does not account for trigger inefficiencies and other hardware effects. The final efficiencies for the signal benchmarks mentioned above are given in appendix A.2.4.

\subsubsection{Validation}

We compare the overall efficiencies, obtained by our simplified calculation, to the total efficiencies given in $[11,23]$. For this purpose, we follow the production prescription in the original analysis by CMS, and generate lepton-like particles by DY processes with CTEQ6L1 PDFs [31]. The ratio of the efficiencies is presented in figure 4(a) for $8 \mathrm{TeV}$, and a relatively good agreement is established. We find that our efficiency and the results by CMS are less than $40 \%$ apart, for all charges for masses larger than $300 \mathrm{GeV}$.

As the cross sections for pair-produced MCHSPs drop sharply with their mass, the final mass bounds are only weakly sensitive to the exact upper limits on the effective cross section. Therefore, inaccuracies in the efficiency estimation would result in much smaller deviations in the mass bounds. The mass bounds resulting from our efficiency calculation are expected to differ from the corresponding bounds calculated with the full detector simulation by less than $10 \%$ for smaller masses, and by much less than $5 \%$ for masses larger than $500 \mathrm{GeV}$. Indeed, as shown in figure 4(b), we were able to reproduce the mass bounds for lepton-like particles with excellent accuracy.

When comparing the efficiencies at the muon trigger level with the values given in [23], we find that other than for $m=100 \mathrm{GeV}$, we overestimate the intermediate efficiency by $5 \%-40 \%$. There are additional effects, not included in our calculation, that might reduce 


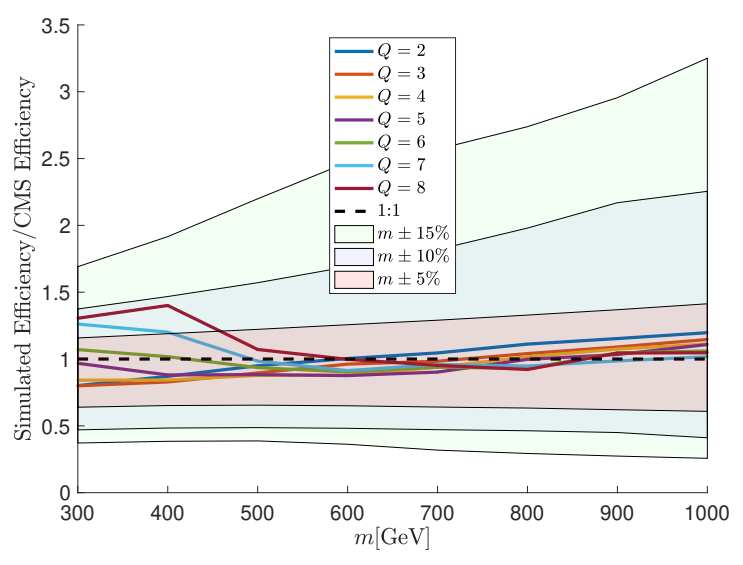

(a)

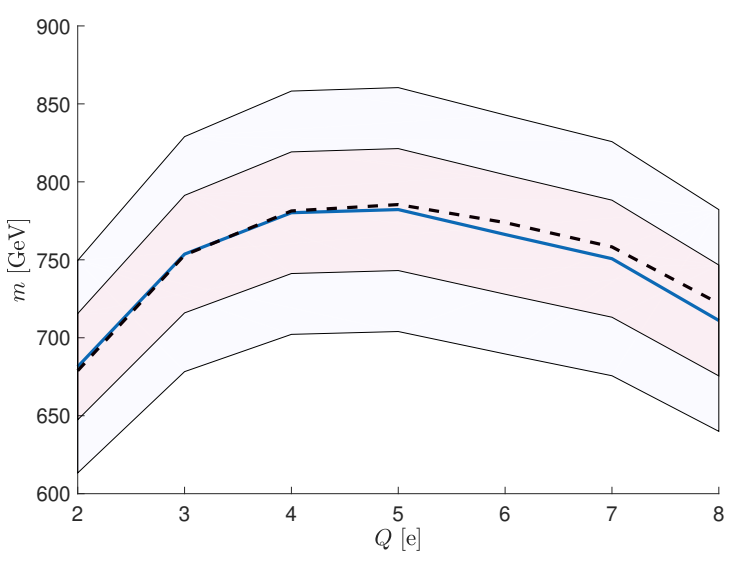

(b)

Figure 4. Simplified efficiency calculation validation. (a) The ratio between our resulting efficiencies and the respective CMS efficiencies for $\sqrt{s}=8 \mathrm{TeV}[11,23]$. Indicated as well are the efficiency deviation bands corresponding to less than $5 \%$ (red), 10\% (light blue) and $15 \%$ (light green) deviation in the mass bound. (b) Reproduced mass bounds for lepton-like particles, following the production mechanism used by CMS. Dashed — the bounds published by CMS [11], using a full detector simulation. Solid - our results using the simplified efficiency calculation. Indicated as well are the $5 \%$ (red) and 10\% (light blue) mass deviation bands, around the our final mass bounds plot.

the number of events passing the muon trigger selection. One such effect is the track reconstruction and matching. Heavy particles with large charges experience large ionization energy loss, and as a result are expected to be less compatible with a global muon pattern. Second, the trigger response and the gaps in the RPC and CSC coverage may increase the distance a candidate must travel to have a sufficient number of hits. Moreover, we do not consider background effects, both from pileup and from hard particles produced in the interaction, that could affect reconstruction. It may also be that we somewhat underestimate the material budget. However, the final selection filters out particles that are too fast, which are favored by the muon trigger. As a result, the overestimation of the muon trigger efficiency could be compensated, and the total efficiency is therefore in agreement with CMS. Even had these effects not canceled out, the final error for the mass bounds would still be smaller than $15 \%$ for masses larger than $500 \mathrm{GeV}$.

\section{Bound state signal at the LHC}

Our second goal is to obtain mass bounds on CTTPs from their signatures as partnerium bound states. In this section, we will discuss the salient features of the partnerium resonance, and introduce our recast procedure, which will be centered around diphoton channel.

The partnerium is unstable due to the annihilation of its constituents, and can be detected as a resonance, with invariant-mass peak at $M \approx 2 m_{\text {partner. }}$ A $J=0$ or $J=2$ partnerium state, made of EM-charged constituents, can always decay through annihilation into $\gamma \gamma, \gamma Z$ and $Z Z$. In the case of the color-triplet CTTPs, it may also decay into a pair of 
gluons. A $J=1$ partnerium, consisting of fermions, can annihilate into $W^{+} W^{-}$[14], or to any SM fermion - anti-fermion pair, through $s$-channel $\gamma / Z$ exchange [5]. Moreover, if the constituent is a top partner, its large coupling to the Higgs implies significant annihilation rates into Higgs pairs and longitudinally polarized Electroweak (EW) gauge bosons (for $J=0$ or 2 partnerium made of scalars), or to $h Z$ (for $J=1$ fermion bound states) [4]. Out of these search channels, the diphoton signal is by far the most sensitive $[4,5]$, especially for the large electric charges we consider. We will thus solely focus on this final state.

Several authors have recast LHC resonance searches to obtain bounds on CTTPs. Mass bounds for scalar and fermion CTTPs of charges $-1 / 3,2 / 3,-4 / 3,5 / 3$ can be inferred from the plots presented in ref. [4]. In addition, the authors of [32] obtained bounds for colored scalars with charges $-7 / 3,8 / 3,-10 / 3$ and of different $\mathrm{SU}(2)_{\text {weak }}$ representations. However, these analyses attributed the dominant partnerium production, binding and decay mechanisms to QCD. This is not necessarily the case for partners with larger charges, as we will see. Ref. [14] contains the only available resonance analysis for charges $1-8$, but is limited to colorless fermions bound in a "Leptonium" [14]. As the leptonium diphoton signal is highly sensitive to the photon PDF, we will also see that a more accurate PDF choice can lead to significantly different conclusions. Thus, similarly to the open-production case, the existing analyses of partnerium-like signatures are insufficient for constraining the parameter space of MCHSPs. We therefore recast a diphoton resonance search, to obtain bounds on the masses of CTTPs and to update the corresponding bounds for lepton-like particles.

Our recast is based on the latest diphoton search, at $\sqrt{s}=13 \mathrm{TeV}$ and an integrated luminosity of $35.9 \mathrm{fb}^{-1}$, published by CMS [33]. As the efficiency of diphoton detection at a given invariant mass is mostly independent of the signal model, we kept it unmodified. We therefore only compute the diphoton production cross section, resulting from a partnerium or a leptonium resonance, accounting for both QCD and EM effects, and using the more precise LUXqed PDF set [15] (see also section 2). The rest of this section is dedicated to the cross section calculation method.

The diphoton resonant production cross section is calculated using the full BreitWigner formula [27]. Thus, we are interested in both the production and the decay channels of the intermediate bound state. The partnerium can be produced by photon-fusion and gluon-fusion (projected onto a color-singlet), regardless of the partner's spin. A leptonium, consisting of color-singlet fermions, can be produced via photon-fusion. A fermion-based bound state can also be produced via DY processes, mediated by a photon or a $Z$ boson [5], however it may not decay into a diphoton final state. The allowed decay channels of a diphoton resonance are those of a $J=0,2$ resonance, discussed above. The resulting diphoton cross section would therefore follow

$$
\begin{aligned}
\sigma_{p p \rightarrow B \rightarrow \gamma \gamma}= & 8 \pi \int_{0}^{1}\left[\frac{1}{64} \mathcal{L}_{g g}(\tau) \Gamma_{B \rightarrow g g}+\mathcal{L}_{\gamma \gamma}(\tau) \Gamma_{B \rightarrow \gamma \gamma}\right] \\
& \times \frac{\Gamma_{B \rightarrow \gamma \gamma}}{\left(\hat{s}-4 m^{2}\right)^{2}+\hat{s}\left(\Gamma_{B \rightarrow \gamma \gamma}\left(1+2 \tan ^{2} \theta_{W}+\tan ^{4} \theta_{W}\right)+\Gamma_{B \rightarrow g g}\right)^{2}} \frac{d \tau}{\tau},
\end{aligned}
$$

where $\tau=\hat{s} / s$, with $\sqrt{\hat{s}}$ being the total partonic COM energy, and $\theta_{W}$ is the weak angle. 
The parton luminosity for a pair of partons $a, b$ is

$$
\mathcal{L}_{a b}(\tau)=\tau \int_{\tau}^{1} \frac{d x}{x} f_{a}(x) f_{b}\left(\frac{\tau}{x}\right)
$$

where $x$ is the fraction of the proton momentum carried by the parton and $f_{a}$ is the PDF of the parton, which we evaluate at the factorization scale $m$. For colorless fermions, the diphoton cross section is the same, excluding QCD contributions [14]. The relevant decay widths for scalar CTTPs are given by [5, 34]

$$
\begin{array}{ll}
\Gamma_{B \rightarrow \gamma \gamma}=\frac{24 \pi \alpha^{2} Q^{4}}{M^{2}}|\Psi(0)|^{2} & (\times 2 \text { for fermions }, \times 1 / 3 \text { for color-singlets }), \\
\Gamma_{B \rightarrow g g}=\frac{16}{3} \frac{\pi \alpha_{s}^{2}}{M^{2}}|\Psi(0)|^{2} & (\times 2 \text { for fermions }),
\end{array}
$$

where $M$ is the mass of the resonance, and modification factors for fermions and for colorsinglet particles are given in parentheses. The naturalness-enhanced decays of the partnerium were found to be negligible when calculating the total decay width.

Colored particles of large charges could have a non-negligible contribution to their binding coming from the EM force

$$
V(r)=-\frac{C \bar{\alpha}_{s}+Q^{2} \alpha}{r}
$$

where $\mathrm{C}$ is the Casimir of $\mathrm{SU}(3)_{c}, C_{3}=4 / 3$ for a color-triplet and $C_{0}=0$ for a color-singlet. The wavefunction at the origin is

$$
|\psi(0)|^{2}=\frac{\left(C \bar{\alpha}_{s}+Q^{2} \alpha\right)^{3} M^{3}}{8 \pi n},
$$

where $n$ is the radial excitation level. Since the contributions from $n \geq 2$ states are negligible, we keep only the ground state contribution [5]. In addition, we only consider the LO effects in the binding potential. The higher order effects have been studied in $[17,35,36]$. They find a noticeable though not dramatic enhancement of the signal cross section. Therefore, our bounds are somewhat conservative. One should note that in the decay rates and in the wavefunction $M^{2} \rightarrow \hat{s}$, as $\hat{s}$ is the mass of the resonance [37].

The decay rates of the partnerium and the leptonium grow significantly with the charge of the constituents. For lepton-like particles, and for CTTPs with large charges, the bound state annihilation rate approaches a $Q^{10}$-dependence, as a result of the dominant EM contributions. Therefore, the diphoton cross section will exhibit high charge sensitivity.

The signal benchmarks are as described for the open-production channel recast. A resonance treatment is indeed appropriate for all the charges we consider, since $\Gamma / M \lesssim 10^{-1}$ for constituents with $Q \lesssim 8$. For CTTPs and lepton-like particles with $Q \leq 4$, we have found that the narrow width approximation is more stable numerically. The production cross section for a narrow $\gamma \gamma$ resonance, via the decay of spin- 0 partnerium bound state 
$B$, is given by

$$
\begin{aligned}
\sigma_{p p \rightarrow B \rightarrow \gamma \gamma}= & \sigma_{p p \rightarrow B} B r_{B \rightarrow \gamma \gamma} \\
= & \frac{\pi^{2}}{m^{3}}\left[\frac{1}{64} \mathcal{L}_{g g}\left(\frac{4 m^{2}}{s}\right) \Gamma_{B \rightarrow g g}+\mathcal{L}_{\gamma \gamma}\left(\frac{4 m^{2}}{s}\right) \Gamma_{B \rightarrow \gamma \gamma}\right] \\
& \times \frac{\Gamma_{B \rightarrow \gamma \gamma}}{\Gamma_{B \rightarrow \gamma \gamma}\left(1+2 \tan ^{2} \theta_{W}+\tan ^{4} \theta_{W}\right)+\Gamma_{B \rightarrow g g}},
\end{aligned}
$$

and in the decay rates and wavefunctions $M^{2} \rightarrow 4 m^{2}$, where $m$ is the mass of the partner.

Following the calculation above, using Mathematica package ManeParse 2.0 [38] with LUXqed PDFs [15] and performing numerical integration using Mathematica, we obtain the diphoton cross sections for differently charged MCHSPs, which can be found in appendix B. The resulting current and future-projected bounds are discussed in sections 4 and 5 .

\section{Current status - recast bounds}

We are now in a position to obtain and compare lower bounds on the masses of MCHSPs from the (recast) searches for their open-production and closed-production signatures. We begin by describing the current mass bounds, corresponding to the latest observations. Our bounds from the most recently published searches are presented in table 3 and compared in figure 5 . Conservatively combining the bounds by taking the stricter one for each signal benchmark, we obtain the current mass bounds at a minimal CL of 95\%, highlighted in the table.

To obtain current constraints on MCHSPs from the open channel, we utilize the most recent search for above-threshold MCHSPs, conducted by CMS at $\sqrt{s}=8 \mathrm{TeV}$ [11]. The limits on particle masses, in a given signal model, are derived by first obtaining a $95 \%$ Confidence Level (CL) upper limit on the effective cross section, and then choosing the mass such that the theoretical effective cross section saturates this limit. Following CMS, we apply a hybrid Bayesian-frequentist p-value computation [39], with the relevant parameters given in the original analysis. Our resulting upper limit is consistent with that inferred from CMS results. The theoretical effective cross sections are calculated by multiplying the cross sections and the efficiencies, as explained in sections 2.1,2.2, and can be found in appendix A.3.

Analogously to the open channel, we derive mass bounds for MCHSPs from their bound state signatures as well. For the closed production case, we require the theoretical diphoton production cross section, induced by the bound state resonance, as explained in section 3 , to saturate the upper limits at 95\%-CL. For the current bound, we employ the CMS limit given in [33] for $\sqrt{s}=13 \mathrm{TeV}$ at $\mathcal{L}=35.9 \mathrm{fb}^{-1}$. It should be noted the signal efficiency in [33] was calculated for gluon-fusion production, and could be slightly different for photonproduced resonances. The experimental bounds on a diphoton resonance in [33] were given for three resonance-width benchmarks: $\Gamma / M=1.4 \cdot 10^{-4}$ (narrow), $\Gamma / M=1.4 \cdot 10^{-2}$ (midwidth) and $\Gamma / M=5.6 \cdot 10^{-2}$ (wide). Therefore, when available, we use narrow resonance bounds for $\Gamma / M \lesssim 5 \cdot 10^{-3}$ ( $Q \lesssim 5$ for color-triplets, $Q \lesssim 6$ for color-singlets), mid-width resonance bounds for $5 \cdot 10^{-3} \lesssim \Gamma / M \lesssim 3 \cdot 10^{-2}(5 \lesssim Q \lesssim 6$ for color-triplets, $6 \lesssim Q \lesssim 7$ 


\begin{tabular}{|c|c|c|c|c|c|c|c|c|}
\hline $\mathrm{Q}[\mathrm{e}]$ & $5 / 3$ & $8 / 3$ & $11 / 3$ & $14 / 3$ & $17 / 3$ & $20 / 3$ & $23 / 3$ & channel \\
\hline \multirow{2}{*}{$\begin{array}{c}\text { color-triplet } \\
\text { scalar }\end{array}$} & 970 & 980 & 980 & 980 & 970 & 950 & 930 & \multirow{2}{*}{$\begin{array}{l}\text { open } \\
\text { closed }\end{array}$} \\
\hline & 570 & 700 & 970 & 1180 & 1460 & 1800 & 2250 & \\
\hline \multirow{2}{*}{$\begin{array}{c}\text { color-triplet } \\
\text { fermion }\end{array}$} & 1200 & 1200 & 1210 & 1200 & 1190 & 1170 & 1160 & \multirow{2}{*}{$\begin{array}{l}\text { open } \\
\text { closed }\end{array}$} \\
\hline & 590 & 860 & 1080 & 1330 & 1640 & 2050 & $2250^{*}$ & \\
\hline $\mathrm{Q}[\mathrm{e}]$ & $-4 / 3$ & $-7 / 3$ & $-10 / 3$ & $-13 / 3$ & $-16 / 3$ & $-19 / 3$ & $-22 / 3$ & channel \\
\hline \multirow{2}{*}{$\begin{array}{c}\text { color-triplet } \\
\text { scalar }\end{array}$} & 960 & 970 & 980 & 980 & 960 & 950 & 930 & \multirow{2}{*}{$\begin{array}{c}\text { open } \\
\text { closed }\end{array}$} \\
\hline & 430 & 620 & 860 & 1100 & 1360 & 1680 & 2070 & \\
\hline \multirow{2}{*}{$\begin{array}{l}\text { color-triplet } \\
\text { fermion }\end{array}$} & 1200 & 1200 & 1200 & 1200 & 1190 & 1170 & 1150 & \multirow{2}{*}{$\begin{array}{c}\text { open } \\
\text { closed }\end{array}$} \\
\hline & 480 & 850 & 1030 & 1210 & 1520 & 1890 & $2250^{*}$ & \\
\hline $\mathbf{Q}[\mathbf{e}]$ & 2 & 3 & 4 & 5 & 6 & 7 & 8 & channel \\
\hline \multirow{2}{*}{$\begin{array}{l}\text { color-singlet } \\
\text { fermion }\end{array}$} & 690 & 780 & 840 & 870 & 890 & 890 & 890 & \multirow{2}{*}{$\begin{array}{l}\text { open } \\
\text { closed }\end{array}$} \\
\hline & - & - & - & 570 & 980 & 1380 & 1710 & \\
\hline
\end{tabular}

Table 3. Current lower bounds on the masses of MCHSPs. The bounds were obtained from the diphoton resonance signatures at $\sqrt{s}=13 \mathrm{TeV}, \mathcal{L}=35.9 \mathrm{fb}^{-1}$ (closed-production channel) and from the MCHSPs signatures at $\sqrt{s}=8 \mathrm{TeV}, \mathcal{L}=18.8 \mathrm{fb}^{-1}$ (open-production channel). The colored cells are the corresponding combined bounds, given by naively taking the stricter bound of the two searches. Blue — scalar CTTPs, red — fermion CTTPs and black — lepton-like particle. Mass bounds are given in GeV. ${ }^{*}$ Fermion CTTPs with $Q=23 / 3,-22 / 3$, are excluded below $2250 \mathrm{GeV}$, however the exact bound could not be inferred from the search. More details in text.

for color-singlets) and wide resonance bounds for $\Gamma / M \gtrsim 3 \cdot 10^{-2}(6 \lesssim Q$ for color-triplets, $7 \lesssim Q$ for color-singlets)

The diphoton cross section limit observed in the search was given up to resonance masses of $4500 \mathrm{GeV}$. However, for colored fermions with $Q>6.9$ the corresponding $\gamma \gamma$ cross section is larger than the observed limit throughout the available mass range. They are thus excluded below $m=2250 \mathrm{GeV}$, but their exact mass bound can not be explicitly inferred from this search.

\subsection{Bounds from open signatures of MCHSPs}

We find that scalar and fermion CTTPs are excluded below masses of roughly $1 \mathrm{TeV}$ and $1.2 \mathrm{TeV}$, respectively. Interestingly, the bounds are almost charge independent both for scalar and fermion CTTPs. As can be seen in figure 6, this is a result of a coincidental balance between the production cross sections and the efficiencies at which color-triplet MCHSPs could be directly observed. On the one hand, the search becomes less efficient as the charge of the particle increases. For smaller masses, this is mainly a result of the $p_{T} / Q$ selection, while for larger masses, the timing requirement, imposed by the muon trigger, becomes more important, due to the particle's large ionization energy loss. On the other hand, the cross sections grow with the charge of the particle. The production rate consists of the Q-independent QCD processes, the $Q^{2}$-dependent $g \gamma$-fusion and EW-mediated DY processes, and the $Q^{4}$-dependent photon-fusion. As we have shown in section 2.1, each 


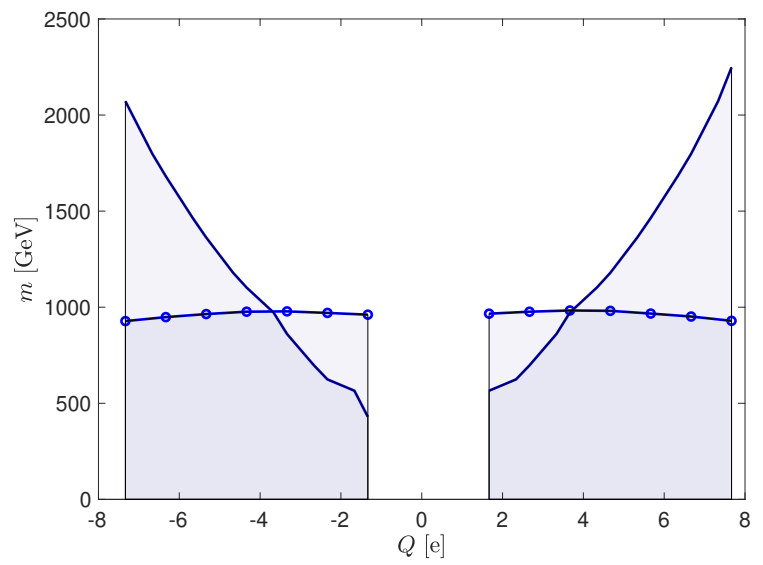

(a) Colored scalars.

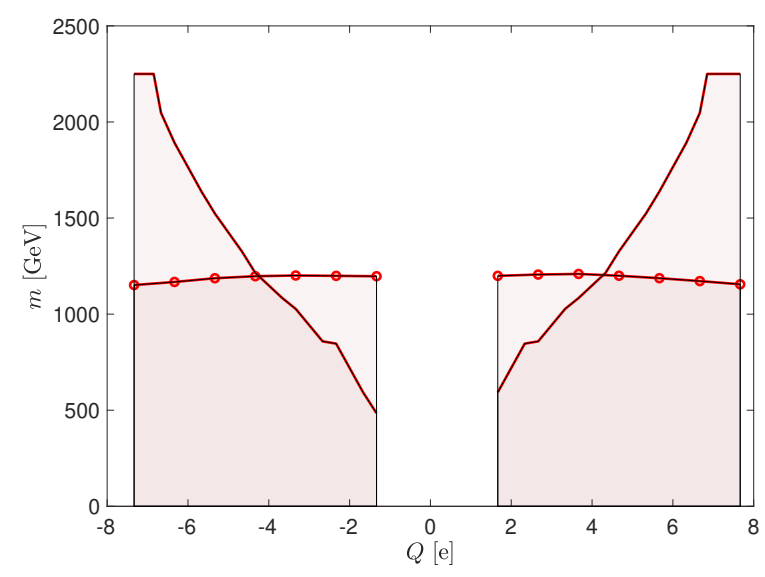

(b) Colored fermions.

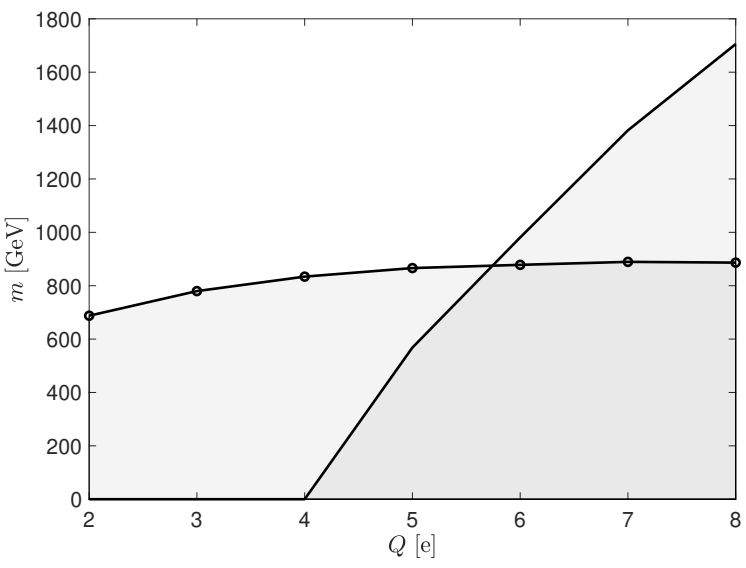

(c) Colorless fermions.

Figure 5. Lower mass bounds, as given by the most recent searches. Solid - a diphoton resonance search at $\sqrt{s}=13 \mathrm{TeV}, \mathcal{L}=35.9 \mathrm{fb}^{-1}$ [33] (closed-production channel). Round markers — a search for MCHSP tracks at $\sqrt{s}=8 \mathrm{TeV}, \mathcal{L}=18.8 \mathrm{fb}^{-1}$ [11] (open-production channel). Shaded - regions excluded by each channel. More details in text. 


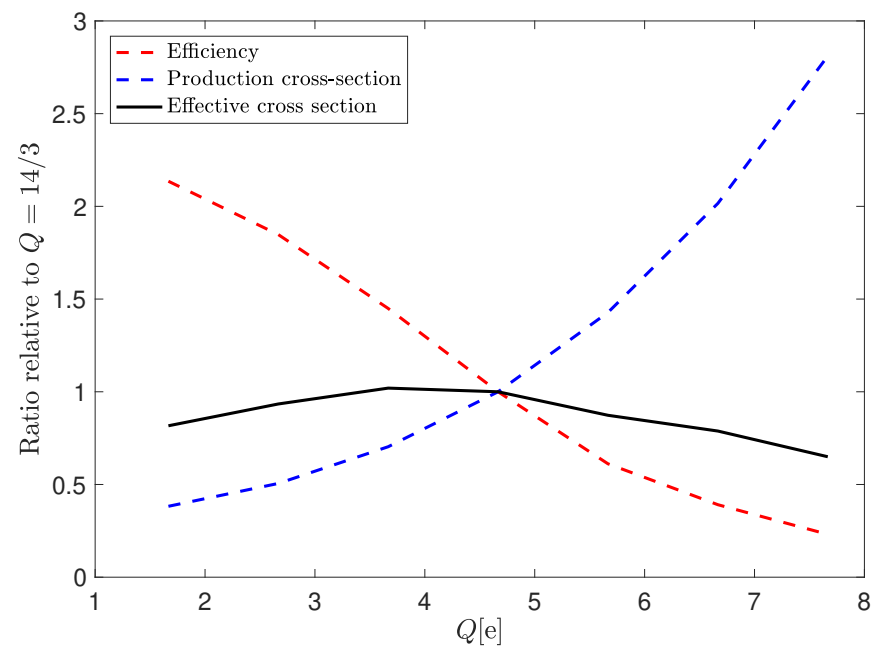

Figure 6. Detection efficiency, production cross section and the resulting effective cross section $\sigma \cdot \epsilon$ for a color-triplet scalar of $m=1000 \mathrm{GeV}$, at $\sqrt{s}=8 \mathrm{TeV}$. All are presented relative to their value for a color-triplet scalar of $Q=14 / 3$.

subprocess becomes dominant at a different mass scale, resulting in a rather strong chargedependence for the production rates of heavy partners. The bounds on the masses of lepton-like particles are slightly more charge dependent. We find colorless fermions to be excluded below a mass of $690 \mathrm{GeV}$ for $Q=2$, and below $890 \mathrm{GeV}$ for $Q=8$. This is a result of the larger charge dependence of the production cross section of lepton-like particles, in the absence of the charge-independent QCD production. Due to hadronization, the bounds in the open channel are asymmetric for positively and negatively charged color-triplets.

\subsection{Bounds from closed signatures of MCHSPs}

The diphoton data excludes color-triplet MCHSPs of charges larger than $\sim 4(\sim 7)$ at masses below $1 \mathrm{TeV}(2 \mathrm{TeV})$. Due to the smaller production and decay rates of bound states consisting of color-singlets, the bounds placed on lepton-like particles are somewhat weaker. Lepton-like particles of charges larger than 5 (8) are excluded below masses of $0.5 \mathrm{TeV}(1.7 \mathrm{TeV})$. The charge dependence of the mass bounds coming from the closedproduction signatures is understandably large, due to the dominant EM effects contributing to production, binding and decay, as explained in section 3. These result in a significant charge dependence of the diphoton resonant cross section, that can be as much as $Q^{10}$ dependent for lepton-like particles. In addition, the efficiency for the diphoton search is not directly related to the bounded constituents charges. The bounds are symmetric for negative and positive charges, as the diphoton cross section in the section 3 is an even function of the $Q$.

\subsection{Combined bounds}

Combining the searches in the open and the closed channels provides powerful constraints on MCHSPs models. As shown above, the current limits derived from the direct search for 


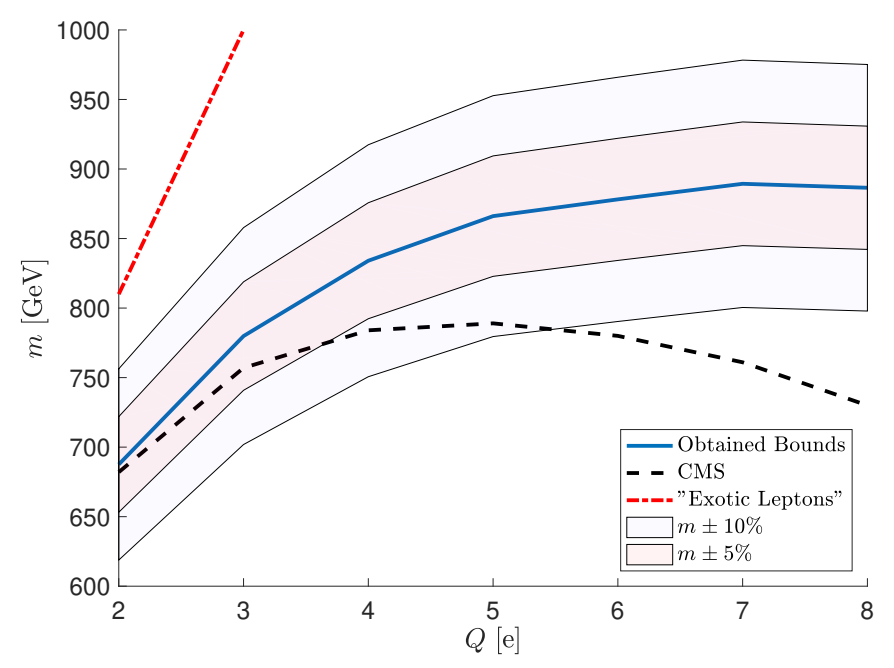

Figure 7. Comparing the lower mass bounds on multiply-charged lepton-like particles, coming from the different analyses of the open-production signature. Dashed - results published by CMS [11]. Dash-dotted red - bounds for $Q=2,3$ given in [14]. Solid blue - mass bounds calculated in this study with $5 \%$ (Red) and 10\% (Light blue) deviation bands.

MCHSPs are stronger for charges smaller than $\sim 4$ for scalar and fermion color-triplets, and for charges smaller than $\sim 6$ for colorless fermions, while for larger charges the diphoton exclusion bounds dominate. Therefore, we benefit from considering both searches, even by naively setting the bound at the larger of the two. Upon further statistical analysis, one should be able to combine the searches as the two channels must be explained simultaneously for stable particles, and thus obtain even stronger mass bounds at 95\% CL.

\subsection{The leptonic case - comparison to the literature}

Since lepton-like particles have been studied in the past, we may now compare our new bounds for lepton-like particles to those found in the literature. As we will see, the bounds we have obtained are in disagreement with the existing results. These differences are mainly a result of our new cross section calculations, which are more exhaustive and reliable, compared to previous analyses.

As shown in figure 7, the mass bounds we have obtained from the open-production signature are stricter than those published by CMS [11]. While the analysis by CMS considered DY-production exclusively, we also include photon-fusion production. Similarly to [14], we find that photo-production processes significantly enhance the cross sections for particles with large charges, and therefore the bounds have strengthened.

The choice of the PDF plays an essential role in calculating the production cross sections, and is particularly important when considering photo-production processes. This can be inferred by comparing our mass bounds, obtained using LUXqed PDFs set, to the bounds presented in [14], derived using NNPDF2.3QED [16], as both analyses considered the same production processes. As can be seen in figure 7, the mass bounds for colorless fermions, derived from our analysis of the open-production channel, are much weaker than the bounds set by the corresponding analysis in [14]. The same trend emerges when 
comparing the closed-production signature analyses, and we find our bounds to be less stringent than those previously obtained in [14]. The origin of these differences can be traced to the choice of the photon PDF. As discussed in [15] (see also [40]), the way the photon PDF is obtained in the NNPDFx.yQED sets is afflicted by large uncertainties. For the $\gamma \gamma$ parton luminosity at invariant masses of $1-3 \mathrm{TeV}$, as relevant to our analysis, the resultant uncertainty can be more than an order of magnitude. The precise extraction of the photon PDF via the method of $[15,18]$, using ep data, implies, via the resulting LUXqed PDF set, a photon luminosity which is as much as a factor of 60 lower than that obtained for central values of the NNPDF2.3QED set. As a result, the cross section calculations in ref. [14], which are based on those central values, substantially overestimate the contributions coming from photon fusion (as well as other photon-induced components) to the cross section. Consequently, the bounds in [14] need to be corrected down to those derived and presented here.

\section{$5 \quad$ Future scenarios — discovery and exclusion}

In order to obtain the prospective mass bounds from LHC searches at $\sqrt{s}=13 \mathrm{TeV}$, we consider integrated luminosities of $36 \mathrm{fb}^{-1}, 100 \mathrm{fb}^{-1}$ (current — July 2018) and $300 \mathrm{fb}^{-1}$ (future). Our projected mass bounds from the two kinds of searches are presented in figure 8.

For the closed-production signatures, projected bounds for integrated luminosities of $100 \mathrm{fb}^{-1}$ and $300 \mathrm{fb}^{-1}$, are calculated using the expected upper limits for ATLAS searches of a photo-produced $J=0$ resonance, as given in [41].

Although the LHC has been running in COM energy of $13 \mathrm{TeV}$ since 2015, MCHSPs search results have yet to be updated. Therefore, for the open-production searches, we calculate the expected effective cross section upper limit at 95\%-CL, under the background hypothesis. The expected number of background events is calculated by scaling the corresponding $\sqrt{s}=8 \mathrm{TeV}$ estimate [11] in two ways — by the luminosity ratio and by the luminosity ratio times the pileup ratio. The latter is more conservative, and perhaps more realistic, as some of the selections and the backgrounds involved may depend not only on the luminosity, but also on the amount of pileup in each run.

Following our analysis, we expect the mass bounds from the open-production searches to improve dramatically with COM energy. For $\sqrt{s}=13 \mathrm{TeV}$, the bounds could reach about $1-1.5 \mathrm{TeV}$ for lepton-like particles, $1.5 \mathrm{TeV}$ for scalar CTTPs, and just under $2 \mathrm{TeV}$ for fermion CTTPs, even when only considering an integrated luminosity of $36 \mathrm{fb}^{-1}$. We therefore believe that a dedicated experimental search for MCHSPs, accounting for the additional properties of colored particles, such as nuclear energy loss and charge change, is very much in need.

We find that the interplay between the searches for MCHSP tracks and the searches for diphoton resonances leads to an effective way to probe the parameter space of these models. We will now present how the searches in the open and the closed channels could be combined to better study MCHSPs in the future. 


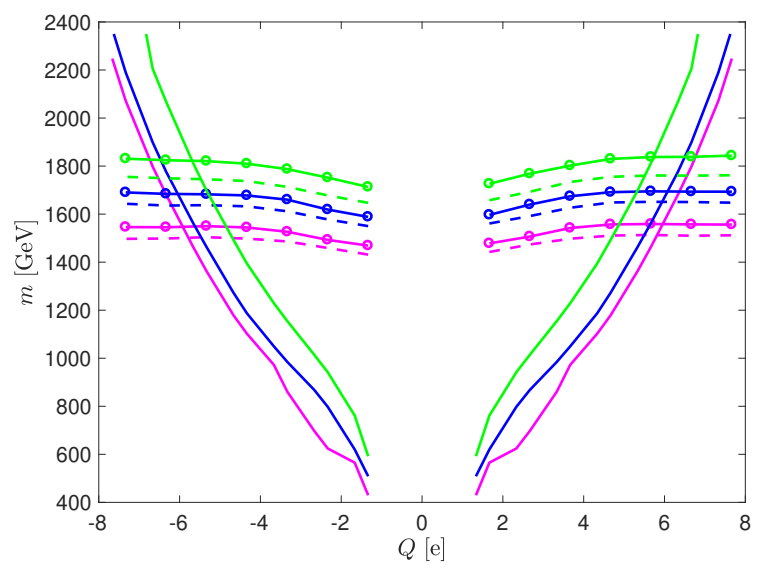

(a) Colored scalars.

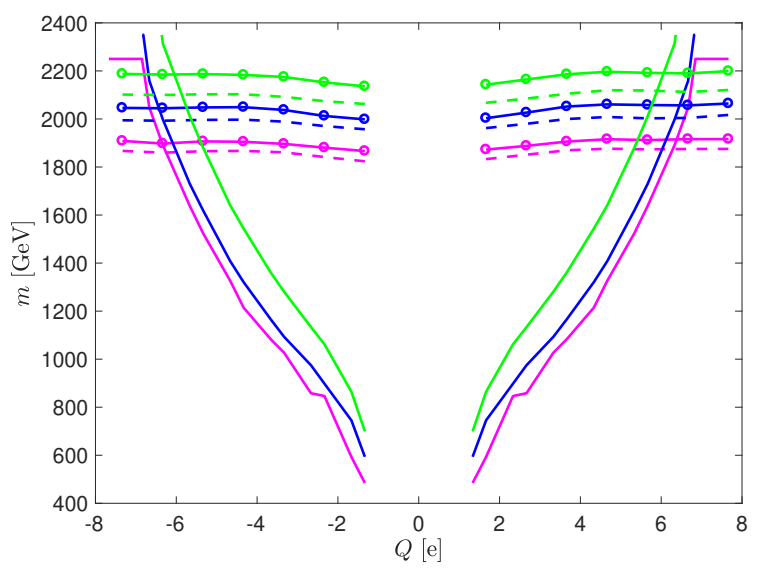

(b) Colored fermions.

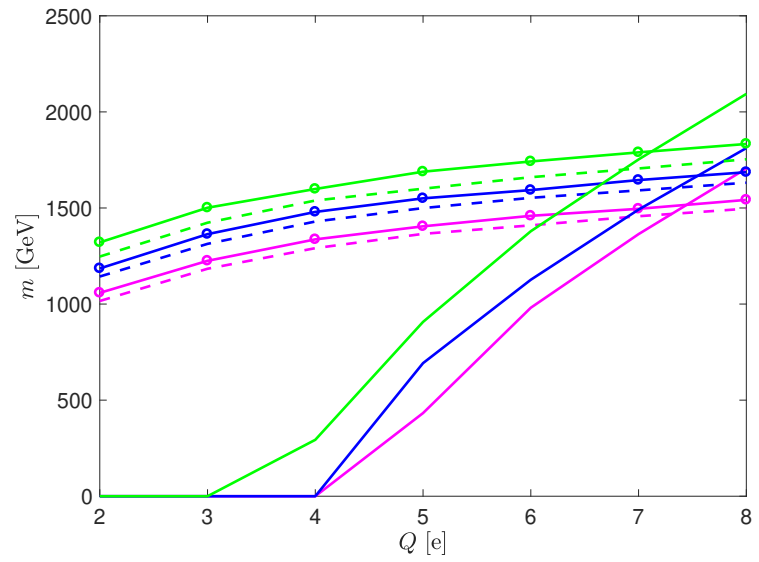

(c) Colorless fermions.

Figure 8. Expected lower mass bounds at $\sqrt{s}=13 \mathrm{TeV}, \mathcal{L}=35.9 \mathrm{fb}^{-1}$ (magenta), $100 \mathrm{fb}^{-1}$ (blue), and $300 \mathrm{fb}^{-1}$ (green). Solid - diphoton resonance searches (closed-production channel). Round markers - searches for MCHSP tracks with luminosity-scaling (open-production channel). Dashed - searches for MCHSP tracks with luminosity and pileup scaling (open-production channel). 
In the case where no excess is observed in both channels, one can combine their results to set upper limits that are significantly stricter than the ones obtained by each search individually. Comparing the two channels assuming the same energy and luminosity, we find that open-production searches are expected to become stronger, and dominate up to charges of about $\sim 6$ for CTTPs, and $\sim 7$ for lepton-like particles. Therefore, these searches are also more likely to carry a potential for discovery. However, in the case of a discovery in the open channel, its analysis might not be able to determine the charge of the observed MCHSP, as we have already established. In addition, the measured kinematics of the particle is different from the truth-level kinematics, due to its unknown charge and ionization energy loss, and will thus be difficult to interpret with good accuracy. On the other hand, given its strong charge-dependence, the diphoton search, although typically less sensitive, can be very useful in breaking the charge degeneracy, or at least in narrowing down the range of allowed charges. The situation could be reversed for very large charges, and the diphoton search could become the discovery channel. In the transition region, correlated excesses in both channels, even if insignificant for each one, may be sufficiently significant to point to a discovery of an MCHSP when combined.

In case of a discovery in both channels, not only would one be able to claim an observation of an MCHSP with higher significance, but also to better study its properties, as we will now demonstrate. First, the mass of the particle could be determined from the diphoton resonance peak. Given the measured mass, one could calculate the theoretical effective cross section, relevant for the open search, and the theoretical diphoton cross section, relevant for the closed search, for MCHSPs of different spins, charges and color representations. As demonstrated for $m=1500 \mathrm{GeV}$ in figure 9, the measurements in both channels would mark a specific point, which could then be related to a specific choice of the particle's quantum numbers. This is true for most of the parameter space, except for the crossing point between a highly charged lepton-like particle and a colored scalar, corresponding to two different choices of quantum numbers. Although measurement uncertainties could make the model distinction less sharp, the appropriate parameter space would be substantially narrowed given the combination of the two measurements.

\section{Conclusions and outlook}

We have studied the LHC phenomenology of MCHSPs. Such particles, that are stable on collider scales and carry exotic electric charges, exist in various extensions of the SM. We introduced the signatures of color-triplet MCHSPs, referred to as CTTPs, which were proposed as a solution to the hierarchy problem [4]. In addition, we reanalyzed the signatures of colorless fermion MCHSPs, referred to as lepton-like particles. We considered both the "closed" channel - where the MCHSP and its anti-particle form a bound state (partnerium/leptonium), detectable as a diphoton resonance, and the "open" channel where each of the MCHSPs propagates approximately independently, detectable in designated searches. For this purpose, we have recast existing analyses, including QCD effects and an updated treatment of EM effects. 


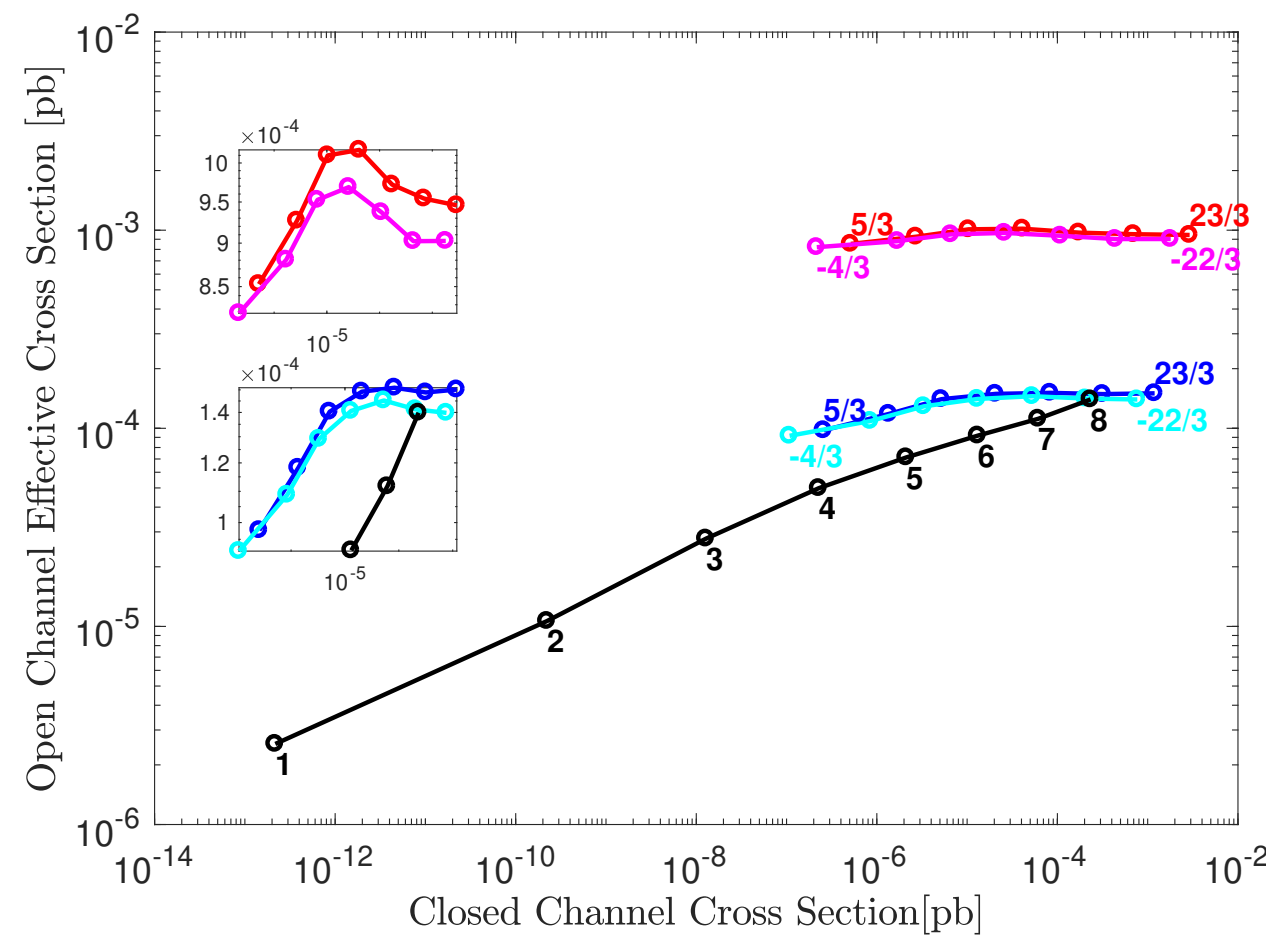

Figure 9. The combined signatures of a hypothetical MCHSP with $m=1500 \mathrm{GeV}$, for different choices of its quantum numbers. In case of a discovery in both channels, combining the observables measured in the two searches could be used to determine the quantum numbers of the newly discovered particle. The lines correspond to different spin-color combinations studied in this work. Black - color-singlet fermions. Blue - color-triplet scalars with positive charges. Cyan - colortriplet scalars with negative charges. Red - color-triplet spin- $1 / 2$ fermions with positive charges. Magenta - color-triplet spin-1/2 fermions with negative charges. Round markers indicate charges spaced by one unit, colored labels indicate the charges. The two subplots on the top-left are magnified views. Top box - negatively-charged and positively-charged color-triplet fermions. Bottom box - negatively-charged and positively-charged color-triplet scalars.

For MCHSPs with relatively small charges, the open-production searches are more important, albeit with only little sensitivity to the charge of the particle. This is in contrast to the diphoton channel, which is more sensitive to MCHSPs with large charges, and exhibits a strong charge-dependence. Thus, a combined search is useful both for the exclusion and for the discovery of MCHSPs. We have obtained bounds on MCHSPs from both production channels, and combined them by taking the more stringent bound for each signal model. We find lower bounds on CTTP masses, that are nearly constant at about $1 \mathrm{TeV}$ for charges $|Q| \leq 4$, then raising to $2.3 \mathrm{TeV}$ at $|Q|=8$. This behavior is due to the closed (diphoton resonance) signature becoming more constraining than open pair production for $|Q| \geq 4$. The bounds on lepton-like particles display an analogous behavior, beginning at about $0.8 \mathrm{TeV}$ and starting to rise at $|Q|=6$, to about $1.7 \mathrm{TeV}$ at $|Q|=8$. The bounds we obtained for lepton-like particles are significantly weaker than those given in [14], but are stronger than the bounds given in [11]. The differences stem from our cross section calculation, which accounts for photo-production processes using LUXqed PDFs set, which is more precise for the photon PDF. 
In addition, we have presented two future scenarios: exclusion and discovery. In the exclusion scenario, where no signal is observed, we have projected the bounds to $13 \mathrm{TeV}$, three integrated luminosities and with or without the pileup scaling. In all cases we find that the bounds become stricter. We therefore strongly encourage a dedicated experimental analysis for MCHSPs, which includes colored particles, and which should combine open production and diphoton resonance signals. ${ }^{2}$ In the event of a discovery, we have shown how combining the measurements at both channels will allow to determine the mass, spin, color, and charge of the observed particle.

In light of our findings, let us briefly comment on the future of open-production searches of MCHSPs. In order to reduce the impact of pileup, both ATLAS and CMS are considering installing a new timing sub-detector, that is capable of measuring TOF at $30 \mathrm{ps}$ resolution [43]. These timing detectors might improve the discovery reach for MCHSPs, by providing an additional, more accurate, discriminator for slow particles. Moreover, they may be able to measure the TOF of a particle prior to its interactions with the material in the calorimeters and in the MS, which are the main cause of ionization energy loss, thus improving detection efficiencies. We leave a dedicated study of the implications of incorporating the information collected by the timing detectors in searches for MCHSPs for future work.

\section{Acknowledgments}

We thank Shikma Bressler for her insights and expertise that greatly assisted the research, and for her comments on the manuscript. We also thank Marumi Kado and Yevgeny Kats for the useful discussions. We are grateful to Luke Arpino, Tom Steudtner, Matthias Schlaffer, Sanmay Ganguly and the WIS LCG team for their kind help with different aspects of the project. The work of GP is supported by grants from the BSF, ERC, ISF, the Minerva Foundation, and the Segre Research Award. GP, SJ and SK acknowledge the support through a Weizmann-UK Making Connections grant, including a studentship jointly funded with the School of Mathematical and Physical Sciences at the University of Sussex. The work of SJ was supported by UK STFC Consolidated Grant ST/P000819/1. SJ and SK thank the Weizmann Institute, and IS the University of Sussex, for the hospitality during visits while working on this project. IS and SK are grateful for STSM support from European Cooperation in Science and Technology (COST) action CA15108 "Connecting Insights in Fundamental Physics".

\footnotetext{
${ }^{2}$ After completing and posting the manuscript, the results of a Run-II ATLAS search for open-production lepton-like particles [42] became publicly available. This is the first LHC analysis corresponding to the $\sqrt{s}=13 \mathrm{TeV}, \mathcal{L}=36 \mathrm{fb}^{-1}$ data in the context of $|Q|>2$ MCHSPs. Similarly to the run-I CMS analysis [11] discussed above, this new ATLAS analysis did not account for photo-production processes. A rough estimate of these effects can be given by recalculating the theoretical production cross sections, as described in our analysis, and comparing them to the cross section upper limits observed by ATLAS to obtain mass bounds. This leads to mass bounds of $1.02 \mathrm{TeV}(Q=2), 1.36 \mathrm{TeV}(Q=5)$ and $1.32 \mathrm{TeV}(Q=7)$, which are in good agreement with our future-projected bounds for the same energy and luminosity. A more precise treatment requires a dedicated efficiency computation, considering the relevant aspects of the ATLAS detector and signal selections, and should be addressed through a reanalysis by ATLAS.
} 


\section{A Open-production signatures}

\section{A.1 Cross sections}

The cross sections for above-threshold pair-production of MCHSPs are presented for $\sqrt{s}=$ $8 \mathrm{TeV}$ and $\sqrt{s}=13 \mathrm{TeV}$ in figures 10 and 11 .

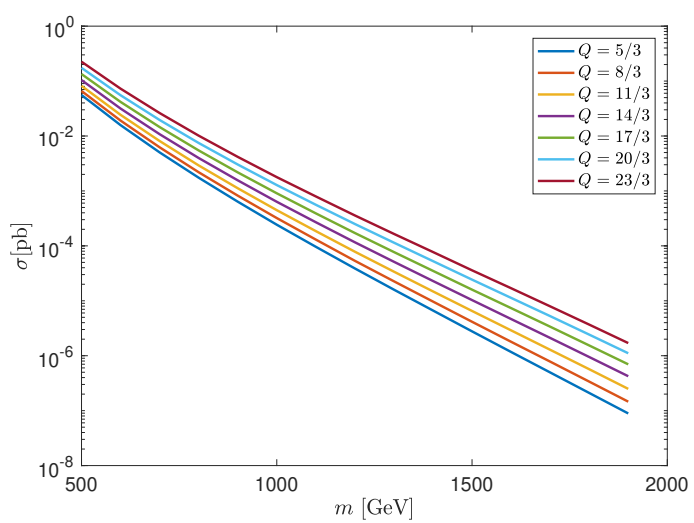

(a) Positvely-charged colored scalars.

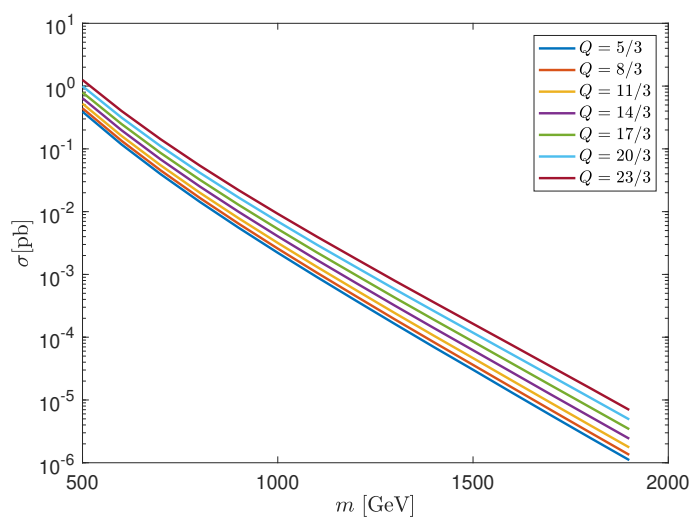

(c) Positvely-charged colored fermions.

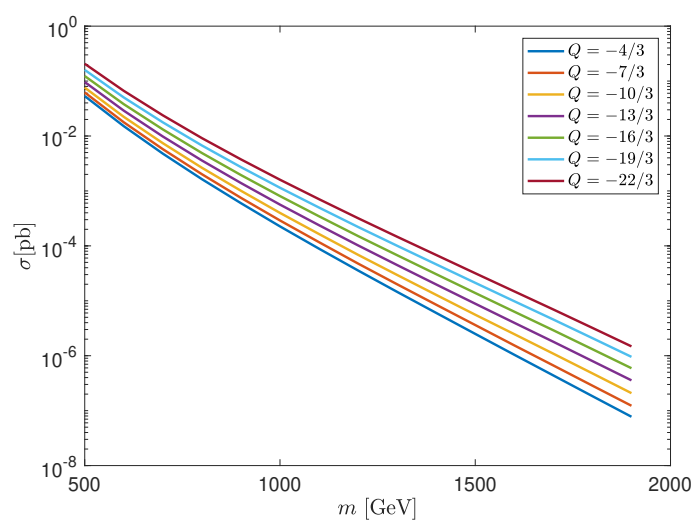

(b) Negatively-charged colored scalars.

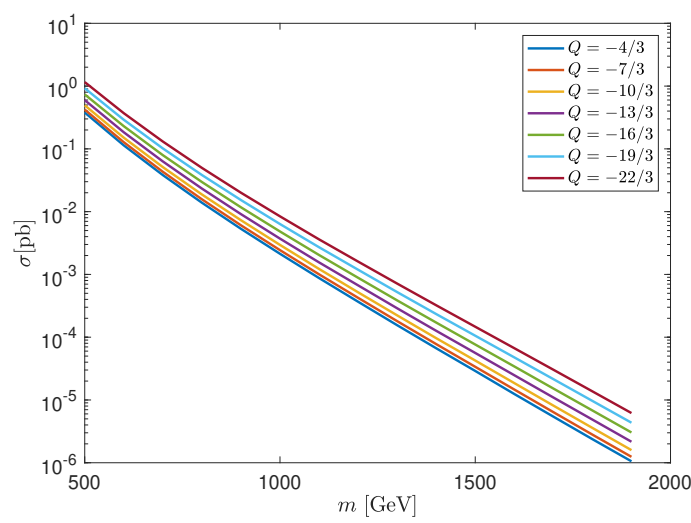

(d) Negatively-charged colored fermions.

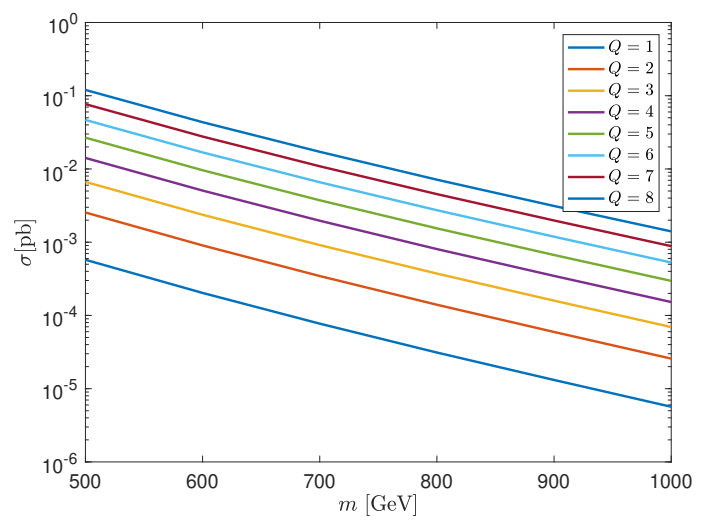

(e) Colorless fermions.

Figure 10. Open-production cross sections at $\sqrt{s}=8 \mathrm{TeV}$. 


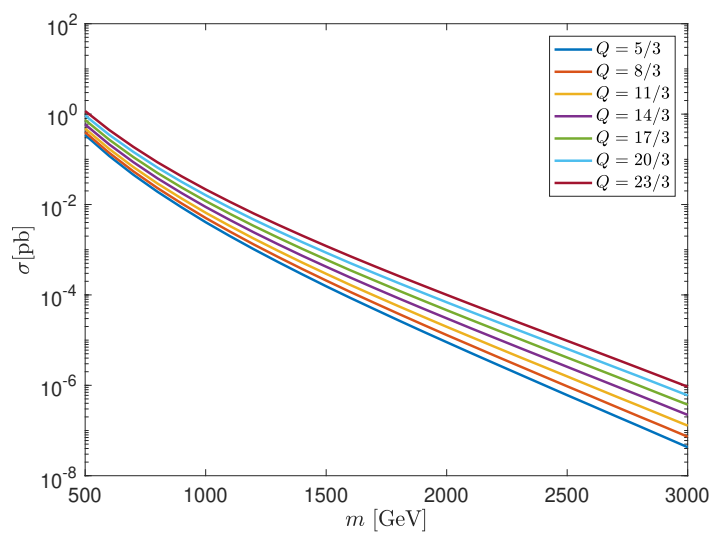

(a) Positvely-charged colored scalars.

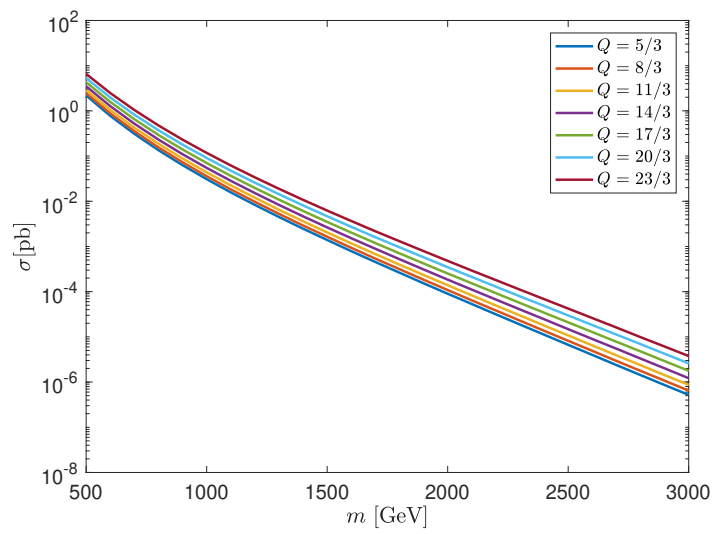

(c) Positvely-charged colored fermions.

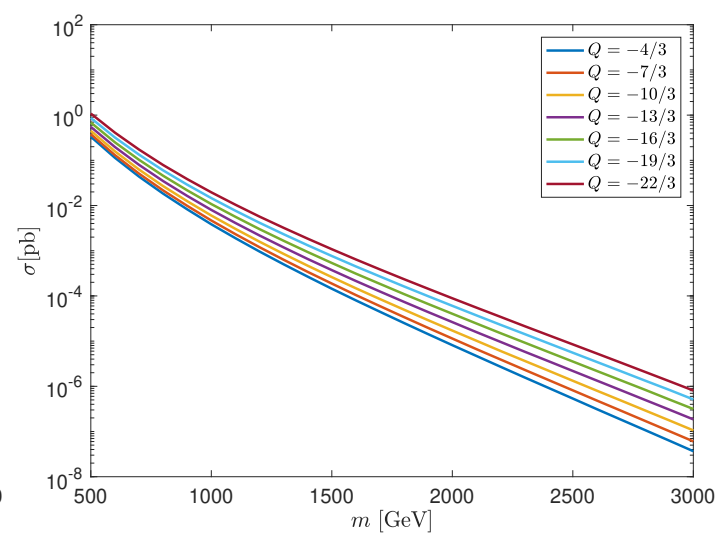

(b) Negatively-charged colored scalars.

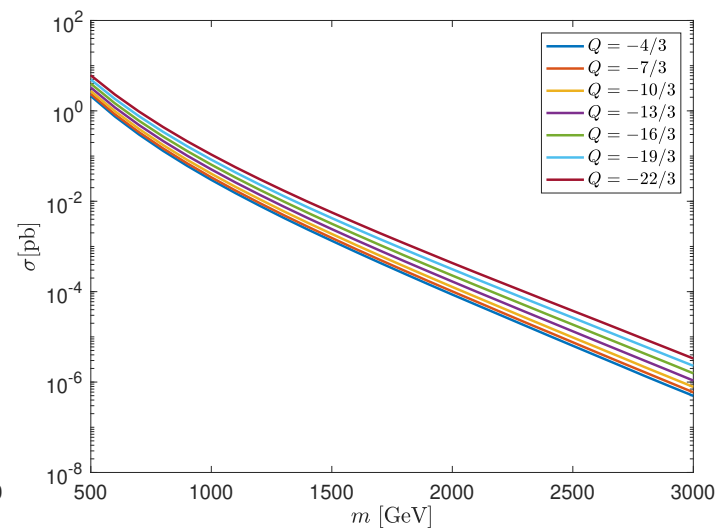

(d) Negatively-charged colored fermions.

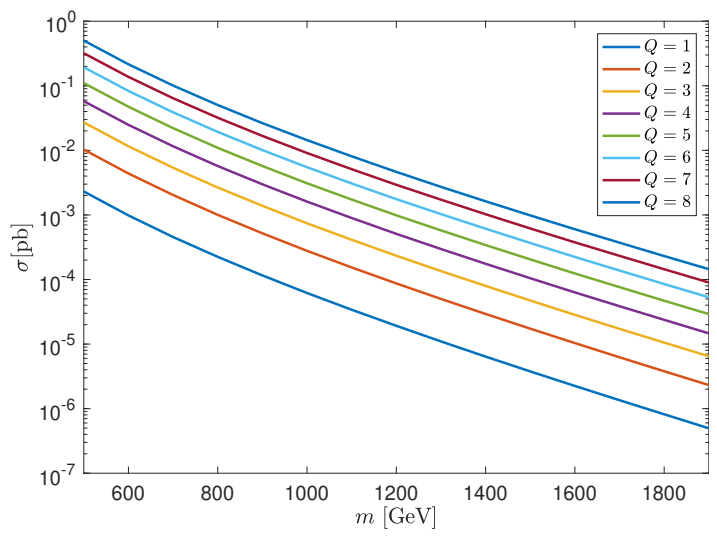

(e) Colorless fermions.

Figure 11. Open-production cross sections at $\sqrt{s}=13 \mathrm{TeV}$. 


\section{A.2 Simplified efficiency calculation}

\section{A.2.1 TOF calculation}

To determine whether a candidate particle is accepted by the muon trigger, we calculate its corresponding TOF by

$$
\begin{aligned}
c \cdot t_{\mathrm{TOF}}= & \frac{\gamma_{0}}{\sqrt{\gamma_{0}^{2}-1}} \cdot x_{\mathrm{HCAL}}^{0}+\int_{0}^{x_{\mathrm{HCAL}}^{f}-x_{\mathrm{HCAL}}^{0}} \frac{\gamma_{\mathrm{Brass}}}{\sqrt{\gamma_{\mathrm{Brass}}^{2}-1}} d x+ \\
& \frac{\gamma_{\mathrm{Brass}}\left(x_{\mathrm{HCAL}}^{f}\right)}{\sqrt{\gamma_{\mathrm{Brass}}\left(x_{\mathrm{HCAL}}^{f}\right)^{2}-1}} \cdot\left(x_{\text {trigger }}-x_{\mathrm{HCAL}}^{f}-\Delta x_{\mathrm{IY}}\right)+\int_{0}^{\Delta x_{\mathrm{IY}}} \frac{\gamma_{\mathrm{Iron}}}{\sqrt{\gamma_{\mathrm{Iron}}^{2}-1}} d x,
\end{aligned}
$$

where $x_{\text {trigger }}$ is the minimal distance a particle must travel, within the trigger time window, in order to be triggered as a muon. As explained in section 2.2, $x_{\text {trigger }}$ is $\eta$-dependent and it is presented in figure. $12(\mathrm{a}) . x_{\mathrm{HCAL}}^{0}, x_{\mathrm{HCAL}}^{f}$ are, respectively - the distance a particle would travel to the entrance and to the exit of the HCAL. The minimal distance a triggering particle would travel in the brass absorber of the HCAL, $x_{\mathrm{HCAL}}^{f}-x_{\mathrm{HCAL}}^{0}$, and in the iron absorber of the iron yoke, $\Delta x_{\mathrm{IY}}$, are also $\eta$-dependent and are shown in figure 12(b). $\gamma(x)$ is the Lorentz factor $\gamma=1 / \sqrt{1-\beta^{2}}$, and it is calculated by numerically solving

$$
\begin{aligned}
& \frac{d \gamma_{\text {Brass }}}{d x}(x)=\frac{Q^{2}}{m} \frac{d E}{d x}{ }_{\text {Brass }}(\gamma), \quad \gamma_{\text {Brass }}(0)=\gamma_{0} \\
& \frac{d \gamma_{\text {Iron }}}{d x}(x)=\frac{Q^{2}}{m} \frac{d E}{d x}{ }_{\text {Iron }}(\gamma), \quad \gamma_{\text {Iron }}(0)=\gamma_{\text {Brass }}\left(x_{\mathrm{HCAL}}^{f}-x_{\mathrm{HCAL}}^{0}\right),
\end{aligned}
$$

where $\gamma_{0}$ is $\gamma$ at production, $Q$ is the charge of the particle and $m$ is the mass of the particle. $d E / d x$ is the energy loss function in the appropriate material for $Q=1$, and is taken from [44] (brass) and [28] (iron).

\section{A.2.2 Straight tracks approximation}

We treat candidates as moving in straight lines, since the bending due to the magnetic field is negligible for particles passing the $p_{T} / Q \geq 40$ selection. A particle tracing a curved track of radius $R$ would travel a distance $l$ in the $r-\theta$ plane before propagating $\Delta r$ in the radial direction, where

$$
\begin{aligned}
l & =R \arcsin \frac{\Delta r}{R}, \\
R & =\frac{p_{T}}{Q \cdot B \cdot 0.303} .
\end{aligned}
$$

The magnetic field in the CMS detector is about $2 \mathrm{~T}$ in the MS and $3.8 \mathrm{~T}$ in the ID [46]. Assuming a maximal $4 \mathrm{~T}$ magnetic field, the $p_{T}$ cut allows minimal $R$ of

$$
R_{\min }=\frac{40}{4 \cdot 0.303} \approx 33.00
$$




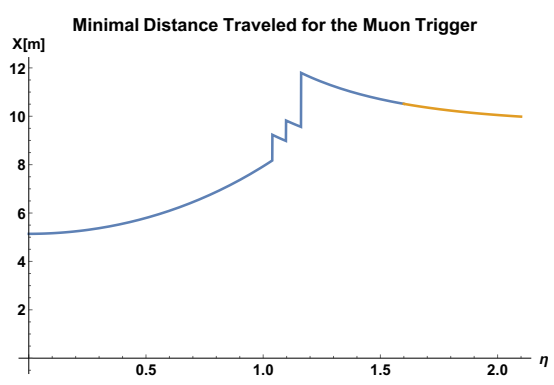

(a)

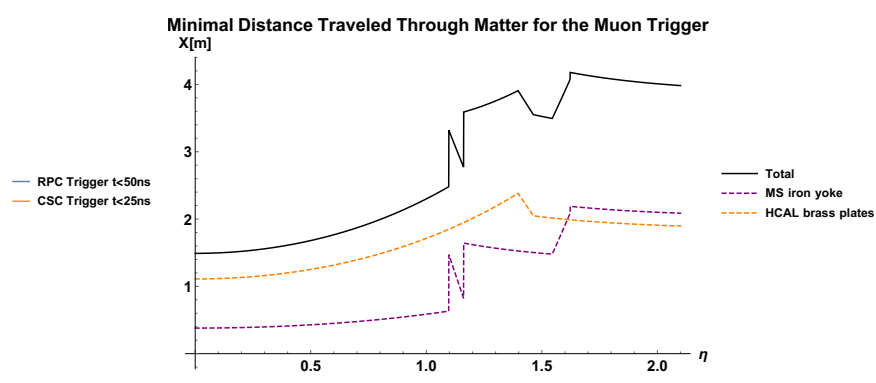

(b)

Figure 12. (a) Minimal distance traveled within the muon trigger time window for high momentum tracks as a function of $\eta$. (b) Distance traveled in matter, relevant for ionization energy loss, within the muon trigger time window as a function of $\eta$. Both (a) and (b) are based on the layout given in [45].

Consider the maximal possible $\Delta r$ distance, which is from the interaction point to the furthest $\mathrm{RPC}$ at $\Delta r_{\max } \approx 7 \mathrm{~m}$,

$$
\left.\frac{l}{\Delta r}\right|_{\max }=\frac{\left.\arcsin \frac{\Delta r}{R}\right|_{\max }}{\left.\frac{\Delta r}{R}\right|_{\max }} \approx 1.0077,
$$

which is indeed a negligible correction to the distance traveled in a straight track.

\section{A.2.3 Global muon offline selection}

In the analysis by CMS, the fraction of particles passing the global-muon selection, relative to the total number of particles produced, is given by

$$
\begin{aligned}
\epsilon_{\text {particles }_{\text {global-muon }}}^{\mathrm{CMS}} & =\epsilon_{\text {online }}^{\mathrm{CMS}} \cdot \epsilon_{\text {offline }}^{\mathrm{CM} \text { global-muon }} \\
& =\frac{\text { Events }^{\mathrm{CMS}}\left(\text { muon-trigger } \cup E_{T}^{\mathrm{miss}}\right)}{\text { Events }} \cdot \epsilon_{\text {offline }_{\text {global-muon }}}^{\mathrm{CMS}}
\end{aligned}
$$

where $\epsilon_{\text {online }}^{\mathrm{CMS}}$ is the fraction of events passing the online selection, relative to the total number of events. $\epsilon_{\text {offline }}^{\mathrm{CMS}}$ is the fraction of particles passing the global-muon criterion, out of the particles passing the online selection. Events is the total number of events and Events ${ }^{\mathrm{CMS}}$ (selection) is the number of events passing a selection. We claim that the particle-level global-muon efficiency can be written as

$$
\begin{aligned}
\epsilon_{\text {particles }_{\text {global-muon }}^{\mathrm{CMS}}} & =\frac{\text { Events }^{\mathrm{CMS}}(\text { muon-trigger })}{\text { Events }} \cdot \frac{\epsilon_{\text {online }}^{\mathrm{CMS}} \cdot \epsilon_{\text {offline }_{\text {global-muon }}^{\mathrm{CMS}}}^{\mathrm{CMS}}}{\epsilon_{\text {events }_{\text {muon-trigger }}}^{\mathrm{CMS}}} \\
& \equiv \alpha^{\mathrm{CMS}}(m, q) \cdot \epsilon_{\text {events }_{\text {muon-trigger }}^{\mathrm{CMS}}}^{\mathrm{CM}}=f(m, q) \cdot \epsilon_{\text {particles }_{\text {muon-trigger }}}^{\mathrm{CMS}},
\end{aligned}
$$

where $\epsilon_{\text {even }}^{\mathrm{CMS}}$

is the fraction of events passing the muon-trigger selection, relative to the total number of events. $\epsilon_{\text {particles }}^{\mathrm{CMS}}$ is the fraction of particles satisfying the muon-trigger requirements, relative to the total number of particles produced, and we hypothesize $f(m, q) \approx 1$. 


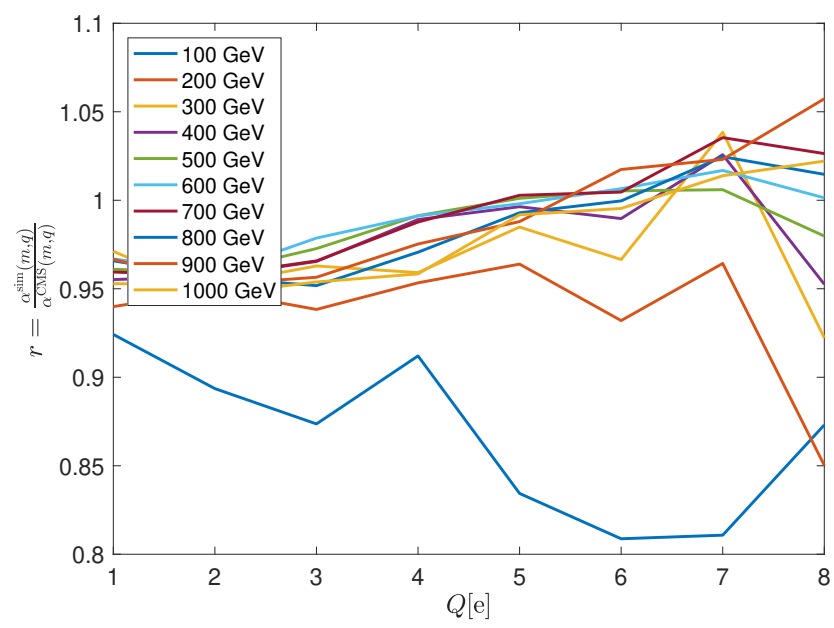

Figure 13. $r=\alpha^{\operatorname{sim}}(m, q) / \alpha^{\mathrm{CMS}}(m, q)$ the ratio of multiplicative factors required to convert the muon trigger event efficiency into the global-muon offline particle efficiency for our procedure, and for CMS.

In our simplified efficiency calculation, we accept only particles that individually satisfy the muon trigger requirements, and omit the global muon selection. So

$$
\begin{aligned}
& \epsilon_{\text {particles }_{\text {global-muon }}^{\text {sim }}}=\frac{\text { Particles }^{\text {sim }}(\text { muon-trigger })}{\text { Particles }} \\
& =\frac{\text { Events }^{\mathrm{sim}}(\text { muon-trigger })}{\text { Events }} \cdot \frac{\epsilon_{\text {particle } s_{\text {muon-trigger }}}^{\text {sim }}}{\epsilon_{\text {events }}^{\text {sim }}} \\
& \equiv \alpha^{\operatorname{sim}}(m, q) \cdot \epsilon_{\mathrm{events}_{\text {muon-trigger }}}^{\mathrm{sim}} .
\end{aligned}
$$

To check the validity of our assumption, independently of our muon-trigger simulation, we calculate the ratio between $\alpha^{\operatorname{sim}}(m, q)$ and $\alpha^{\mathrm{CMS}}(m, q)$

$$
r=\frac{\alpha^{\operatorname{sim}}(m, q)}{\alpha^{\mathrm{CMS}}(m, q)}=\frac{\frac{\epsilon_{\text {particles }}^{\text {sim }} \text { muon-trigger }}{\epsilon_{\text {events }}^{\text {sim }}}}{\frac{\epsilon_{\text {onlinen-trigger }}^{\mathrm{CMS}} \cdot \epsilon_{\text {offline }}^{\mathrm{CMS}}}{\epsilon_{\text {evobal-muon }}^{\mathrm{CMS}}}} .
$$

where the $\epsilon^{\mathrm{CMS}}$ efficiencies are taken from [23], and $\epsilon^{\text {sim }}$ efficiencies are obtained from our calculation. Indeed, as seen in figure $13, r \approx 1$ for all masses and charges for $\sqrt{s}=8 \mathrm{TeV}$. Therefore, we conclude that accepting only particles passing the muon-trigger requirements to be subject for further selection is a reasonable approximation for $\sqrt{s}=8 \mathrm{TeV}$.

\section{A.2.4 Efficiency values}

Here we list the final efficiencies, resulting from our simplified calculation described in section 2.2. The values for a $\sqrt{s}=8 \mathrm{TeV}$ search are given for color-triplet scalars, colortriplet fermions and color-singlet fermions in tables 4,5 and 6 , respectively. The values for a future search at $\sqrt{s}=13 \mathrm{TeV}$ are given in tables 7, 8 and 9, respectively. Masses are in units of $\mathrm{GeV}$. 


\begin{tabular}{|c|ccccccccccccccc|}
\hline$m / Q$ & $-\frac{22}{3}$ & $-\frac{19}{3}$ & $-\frac{16}{3}$ & $-\frac{13}{3}$ & $-\frac{10}{3}$ & $-\frac{7}{3}$ & $-\frac{4}{3}$ & $\frac{5}{3}$ & $\frac{8}{3}$ & $\frac{11}{3}$ & $\frac{14}{3}$ & $\frac{17}{3}$ & $\frac{20}{3}$ & $\frac{23}{3}$ \\
\hline 500 & 0.043 & 0.071 & 0.13 & 0.22 & 0.34 & 0.46 & 0.55 & 0.55 & 0.44 & 0.32 & 0.20 & 0.12 & 0.072 & 0.038 \\
600 & 0.055 & 0.091 & 0.16 & 0.25 & 0.39 & 0.50 & 0.59 & 0.58 & 0.50 & 0.36 & 0.23 & 0.14 & 0.084 & 0.049 \\
700 & 0.065 & 0.11 & 0.17 & 0.28 & 0.41 & 0.54 & 0.61 & 0.60 & 0.52 & 0.39 & 0.26 & 0.17 & 0.097 & 0.059 \\
800 & 0.071 & 0.11 & 0.19 & 0.30 & 0.43 & 0.55 & 0.63 & 0.62 & 0.53 & 0.41 & 0.28 & 0.17 & 0.10 & 0.067 \\
900 & 0.076 & 0.12 & 0.20 & 0.31 & 0.44 & 0.56 & 0.65 & 0.64 & 0.54 & 0.42 & 0.29 & 0.18 & 0.11 & 0.069 \\
1000 & 0.074 & 0.12 & 0.20 & 0.32 & 0.45 & 0.56 & 0.63 & 0.63 & 0.54 & 0.42 & 0.29 & 0.18 & 0.11 & 0.068 \\
1100 & 0.073 & 0.12 & 0.20 & 0.31 & 0.45 & 0.57 & 0.65 & 0.64 & 0.56 & 0.43 & 0.30 & 0.19 & 0.11 & 0.065 \\
1200 & 0.074 & 0.12 & 0.20 & 0.31 & 0.45 & 0.57 & 0.65 & 0.65 & 0.55 & 0.43 & 0.29 & 0.18 & 0.11 & 0.066 \\
1300 & 0.070 & 0.12 & 0.19 & 0.31 & 0.45 & 0.56 & 0.66 & 0.64 & 0.55 & 0.42 & 0.29 & 0.18 & 0.11 & 0.062 \\
1400 & 0.067 & 0.11 & 0.19 & 0.31 & 0.45 & 0.57 & 0.66 & 0.65 & 0.55 & 0.43 & 0.28 & 0.17 & 0.099 & 0.058 \\
1500 & 0.059 & 0.10 & 0.18 & 0.29 & 0.44 & 0.56 & 0.65 & 0.63 & 0.54 & 0.40 & 0.28 & 0.16 & 0.090 & 0.055 \\
1600 & 0.054 & 0.094 & 0.17 & 0.28 & 0.42 & 0.56 & 0.65 & 0.63 & 0.53 & 0.40 & 0.26 & 0.15 & 0.087 & 0.048 \\
1700 & 0.047 & 0.087 & 0.16 & 0.27 & 0.41 & 0.55 & 0.64 & 0.63 & 0.52 & 0.39 & 0.25 & 0.14 & 0.079 & 0.041 \\
1800 & 0.040 & 0.079 & 0.15 & 0.26 & 0.40 & 0.53 & 0.64 & 0.61 & 0.52 & 0.38 & 0.24 & 0.13 & 0.071 & 0.040 \\
1900 & 0.039 & 0.072 & 0.13 & 0.25 & 0.39 & 0.52 & 0.63 & 0.61 & 0.50 & 0.37 & 0.23 & 0.12 & 0.062 & 0.035 \\
\hline
\end{tabular}

Table 4. Efficiencies for color-triplet scalars at $\sqrt{s}=8 \mathrm{TeV}$.

\begin{tabular}{|c|cccccccccccccc|}
\hline$m / Q$ & $-\frac{22}{3}$ & $-\frac{19}{3}$ & $-\frac{16}{3}$ & $-\frac{13}{3}$ & $-\frac{10}{3}$ & $-\frac{7}{3}$ & $-\frac{4}{3}$ & $\frac{5}{3}$ & $\frac{8}{3}$ & $\frac{11}{3}$ & $\frac{14}{3}$ & $\frac{17}{3}$ & $\frac{20}{3}$ & $\frac{23}{3}$ \\
\hline 500 & 0.049 & 0.084 & 0.15 & 0.24 & 0.36 & 0.47 & 0.55 & 0.54 & 0.46 & 0.34 & 0.23 & 0.14 & 0.077 & 0.045 \\
600 & 0.067 & 0.11 & 0.17 & 0.27 & 0.40 & 0.51 & 0.59 & 0.58 & 0.49 & 0.38 & 0.26 & 0.16 & 0.099 & 0.058 \\
700 & 0.076 & 0.12 & 0.19 & 0.29 & 0.42 & 0.54 & 0.61 & 0.60 & 0.52 & 0.41 & 0.28 & 0.18 & 0.12 & 0.075 \\
800 & 0.084 & 0.13 & 0.21 & 0.32 & 0.44 & 0.55 & 0.62 & 0.61 & 0.53 & 0.43 & 0.30 & 0.20 & 0.12 & 0.081 \\
900 & 0.092 & 0.15 & 0.22 & 0.33 & 0.46 & 0.55 & 0.63 & 0.63 & 0.55 & 0.43 & 0.31 & 0.21 & 0.13 & 0.086 \\
1000 & 0.094 & 0.14 & 0.22 & 0.33 & 0.45 & 0.55 & 0.62 & 0.61 & 0.53 & 0.44 & 0.31 & 0.21 & 0.14 & 0.087 \\
1100 & 0.094 & 0.14 & 0.23 & 0.33 & 0.46 & 0.55 & 0.63 & 0.62 & 0.55 & 0.44 & 0.32 & 0.21 & 0.14 & 0.085 \\
1200 & 0.089 & 0.14 & 0.23 & 0.34 & 0.46 & 0.56 & 0.62 & 0.62 & 0.55 & 0.45 & 0.32 & 0.21 & 0.13 & 0.087 \\
1300 & 0.088 & 0.14 & 0.21 & 0.33 & 0.46 & 0.55 & 0.63 & 0.62 & 0.55 & 0.44 & 0.32 & 0.21 & 0.14 & 0.083 \\
1400 & 0.085 & 0.13 & 0.22 & 0.33 & 0.46 & 0.55 & 0.62 & 0.60 & 0.54 & 0.43 & 0.31 & 0.21 & 0.12 & 0.081 \\
1500 & 0.079 & 0.13 & 0.21 & 0.32 & 0.44 & 0.55 & 0.61 & 0.61 & 0.54 & 0.43 & 0.30 & 0.20 & 0.12 & 0.075 \\
1600 & 0.074 & 0.12 & 0.20 & 0.31 & 0.44 & 0.53 & 0.61 & 0.60 & 0.52 & 0.41 & 0.30 & 0.19 & 0.11 & 0.070 \\
1700 & 0.069 & 0.11 & 0.19 & 0.30 & 0.43 & 0.53 & 0.59 & 0.59 & 0.51 & 0.40 & 0.28 & 0.18 & 0.11 & 0.062 \\
1800 & 0.062 & 0.11 & 0.18 & 0.29 & 0.41 & 0.51 & 0.58 & 0.57 & 0.50 & 0.40 & 0.27 & 0.16 & 0.10 & 0.054 \\
1900 & 0.055 & 0.10 & 0.17 & 0.27 & 0.40 & 0.50 & 0.56 & 0.57 & 0.49 & 0.38 & 0.26 & 0.15 & 0.089 & 0.049 \\
\hline
\end{tabular}

Table 5. Efficiencies for color-triplet fermions at $\sqrt{s}=8 \mathrm{TeV}$. 


\begin{tabular}{|c|cccccccc|}
\hline $\mathrm{m} / \mathrm{Q}$ & 1 & 2 & 3 & 4 & 5 & 6 & 7 & 8 \\
\hline 500 & 0.61 & 0.53 & 0.42 & 0.28 & 0.18 & 0.11 & 0.061 & 0.039 \\
600 & 0.65 & 0.58 & 0.47 & 0.33 & 0.21 & 0.13 & 0.080 & 0.049 \\
700 & 0.67 & 0.60 & 0.50 & 0.36 & 0.23 & 0.15 & 0.095 & 0.061 \\
800 & 0.69 & 0.62 & 0.52 & 0.39 & 0.26 & 0.16 & 0.11 & 0.067 \\
900 & 0.69 & 0.62 & 0.53 & 0.40 & 0.27 & 0.17 & 0.11 & 0.071 \\
1000 & 0.68 & 0.60 & 0.53 & 0.41 & 0.28 & 0.17 & 0.11 & 0.072 \\
\hline
\end{tabular}

Table 6. Efficiencies for color-singlet fermions at $\sqrt{s}=8 \mathrm{TeV}$.

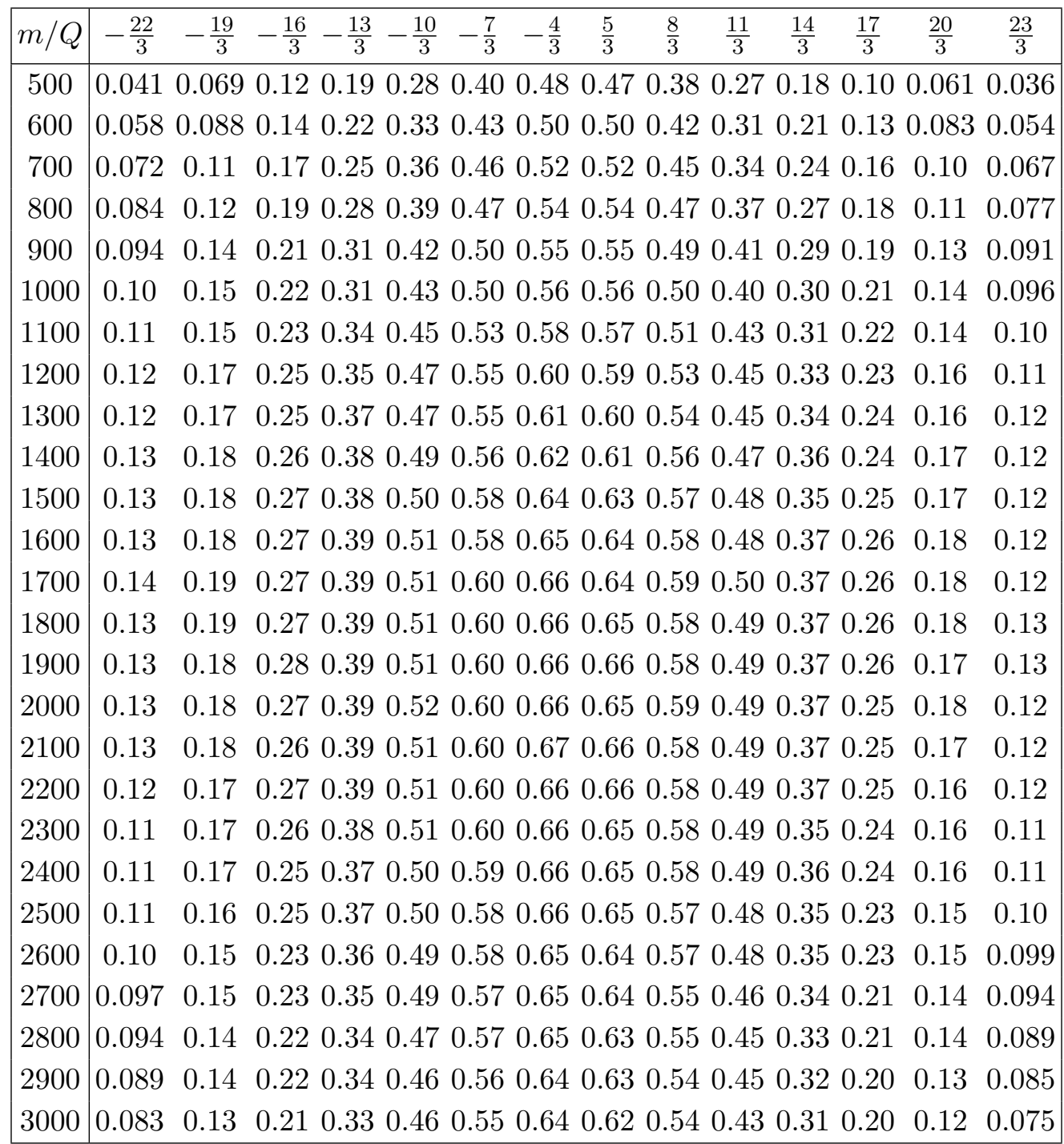

Table 7. Efficiencies for color-triplet scalars at $\sqrt{s}=13 \mathrm{TeV}$. 


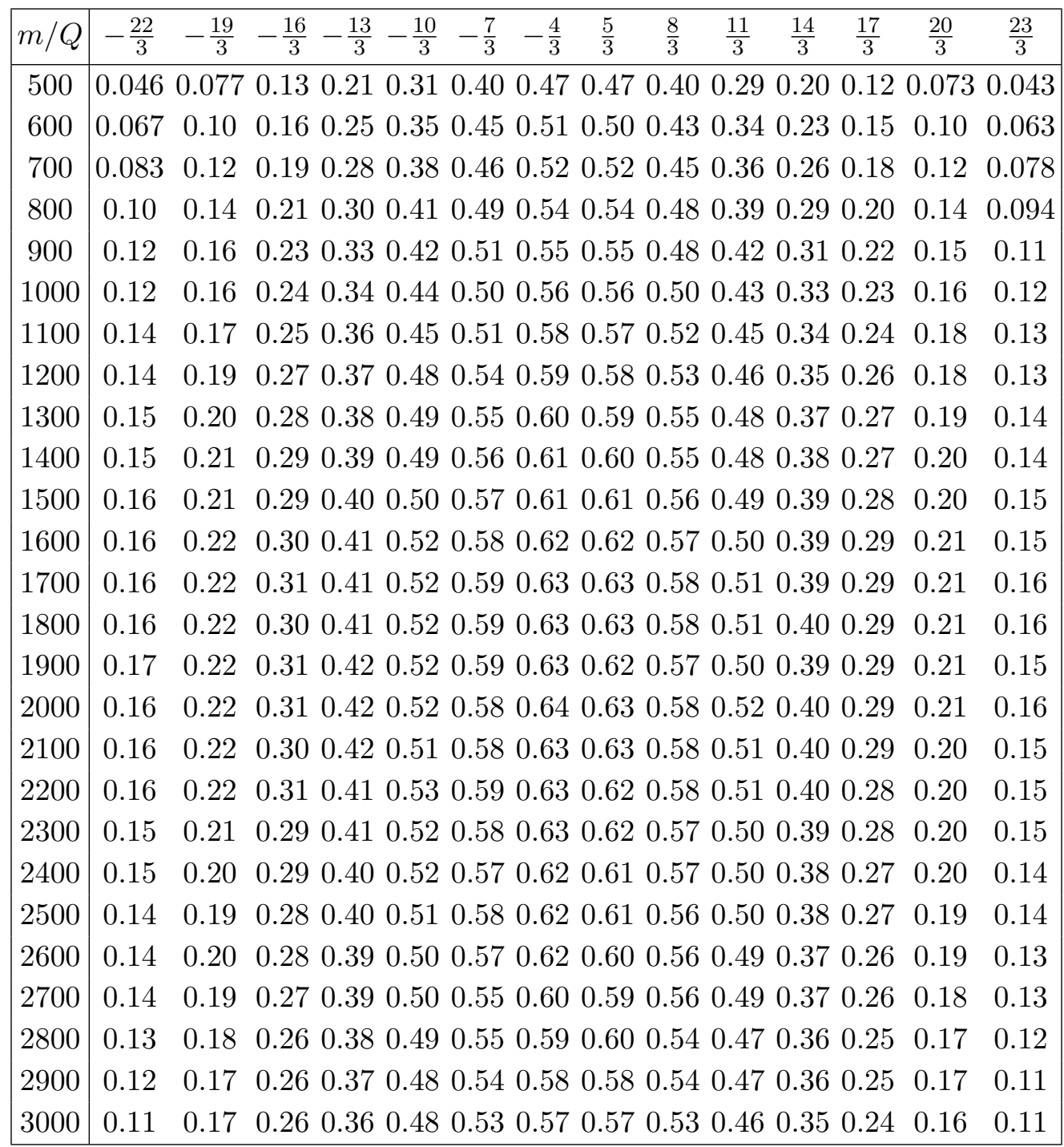

Table 8. Efficiencies for color-triplet fermions at $\sqrt{s}=13 \mathrm{TeV}$. 


\begin{tabular}{|c|cccccccc|}
\hline $\mathrm{m} / \mathrm{Q}$ & 1 & 2 & 3 & 4 & 5 & 6 & 7 & 8 \\
\hline 500 & 0.49 & 0.44 & 0.35 & 0.25 & 0.16 & 0.10 & 0.067 & 0.041 \\
600 & 0.54 & 0.49 & 0.40 & 0.29 & 0.19 & 0.13 & 0.086 & 0.055 \\
700 & 0.56 & 0.52 & 0.44 & 0.32 & 0.22 & 0.15 & 0.10 & 0.066 \\
800 & 0.59 & 0.53 & 0.46 & 0.36 & 0.26 & 0.17 & 0.12 & 0.082 \\
900 & 0.61 & 0.55 & 0.49 & 0.38 & 0.28 & 0.19 & 0.13 & 0.095 \\
1000 & 0.61 & 0.55 & 0.51 & 0.41 & 0.29 & 0.20 & 0.14 & 0.10 \\
1100 & 0.64 & 0.57 & 0.53 & 0.42 & 0.31 & 0.21 & 0.15 & 0.11 \\
1200 & 0.65 & 0.58 & 0.55 & 0.44 & 0.33 & 0.23 & 0.16 & 0.12 \\
1300 & 0.66 & 0.60 & 0.56 & 0.45 & 0.33 & 0.24 & 0.17 & 0.13 \\
1400 & 0.67 & 0.60 & 0.57 & 0.46 & 0.34 & 0.24 & 0.18 & 0.13 \\
1500 & 0.68 & 0.61 & 0.59 & 0.48 & 0.35 & 0.25 & 0.18 & 0.14 \\
1600 & 0.66 & 0.60 & 0.56 & 0.45 & 0.35 & 0.26 & 0.19 & 0.15 \\
1700 & 0.66 & 0.60 & 0.57 & 0.47 & 0.36 & 0.26 & 0.19 & 0.15 \\
1800 & 0.66 & 0.61 & 0.57 & 0.47 & 0.36 & 0.25 & 0.19 & 0.15 \\
1900 & 0.67 & 0.61 & 0.57 & 0.47 & 0.36 & 0.26 & 0.20 & 0.15 \\
\hline
\end{tabular}

Table 9. Efficiencies for color-singlet fermions at $\sqrt{s}=13 \mathrm{TeV}$.

\section{A.3 Effective cross sections}

The effective cross sections for MCHSPs, obtained as a product of the cross sections and the efficiencies corresponding to open-production searches, are presented together with the observed upper limit for $\sqrt{s}=8 \mathrm{TeV}$, and the projected upper limits for $\sqrt{s}=13 \mathrm{TeV}$, in figures 14 and 15. 


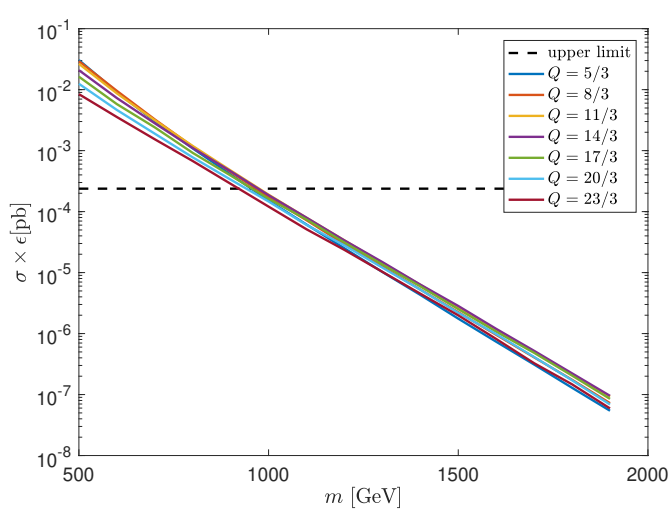

(a) Positvely-charged colored scalars.

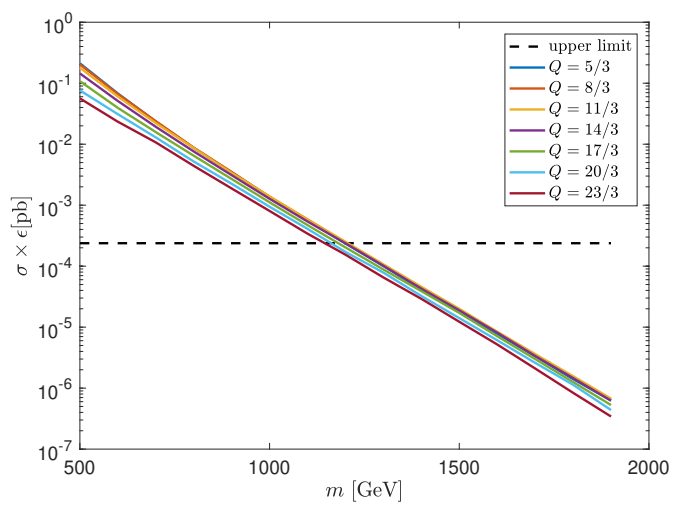

(c) Positvely-charged colored fermions.

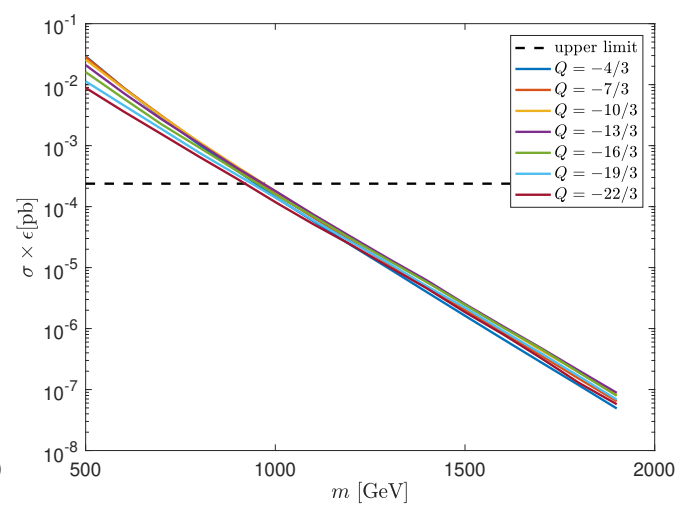

(b) Negatively-charged colored scalars.

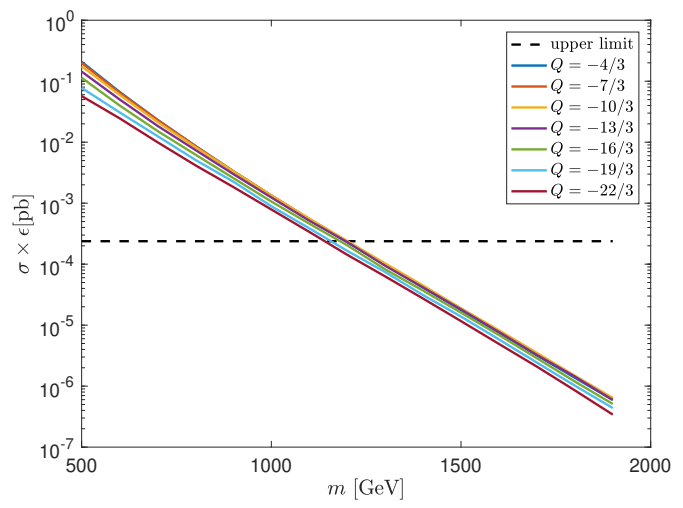

(d) Negatively-charged colored fermions.

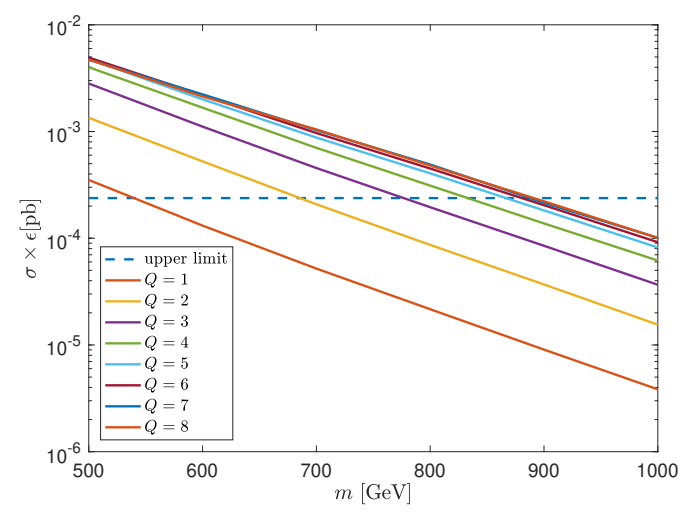

(e) Colorless fermions.

Figure 14. Open-production channel signatures. Effective cross sections $\sigma \cdot \epsilon$ for CMS search [11] at $\sqrt{s}=8 \mathrm{TeV}$, together with the observed upper bound. Solid - theoretical effective cross sections, dashed - observed limit. 


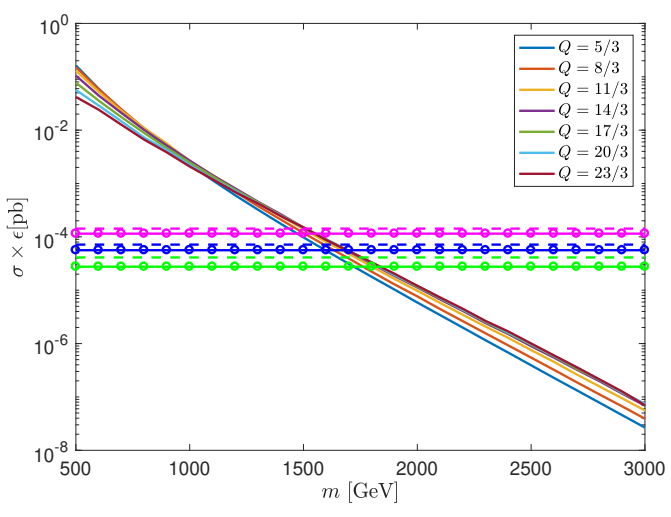

(a) Positvely-charged colored scalars.

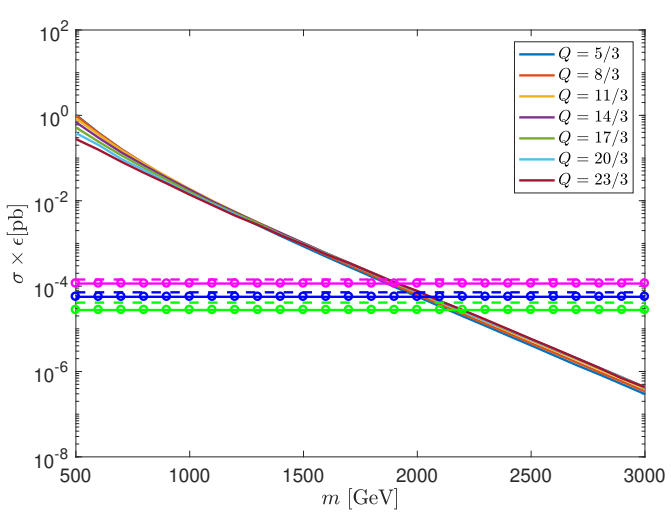

(c) Positvely-charged colored fermions.

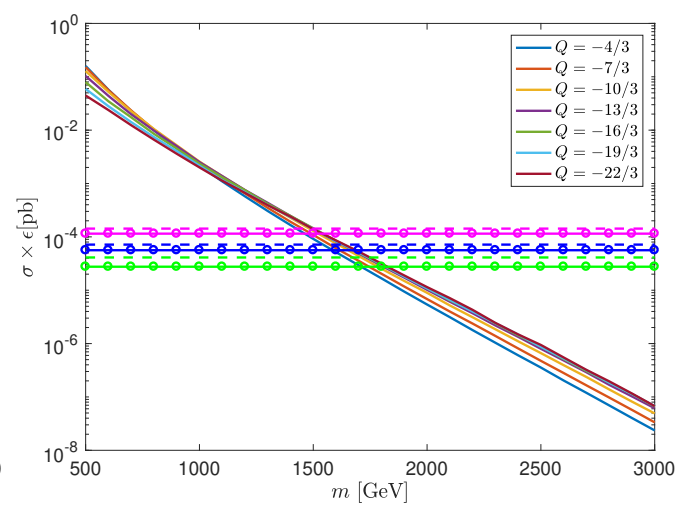

(b) Negatively-charged colored scalars.

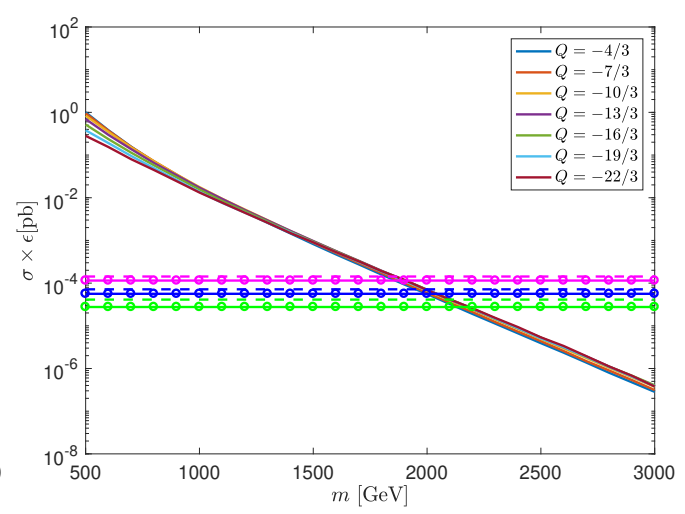

(d) Negatively-charged colored fermions.

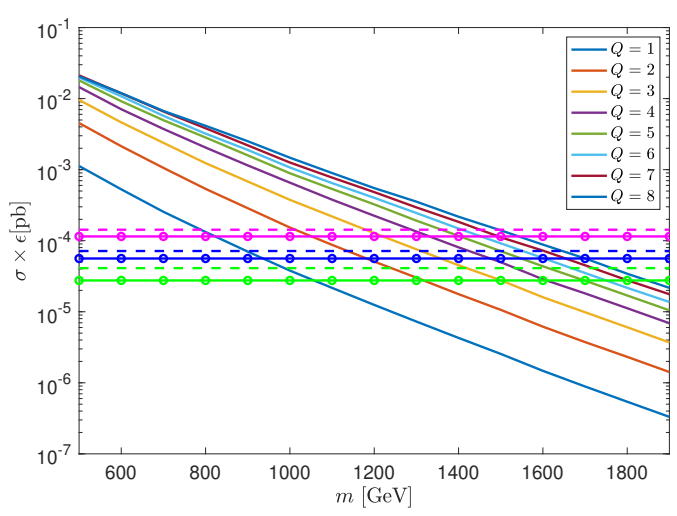

(e) Colorless fermions.

Figure 15. Open-production channel signatures. Effective cross sections $\sigma \cdot \epsilon$ for future CMS searches at $\sqrt{s}=13 \mathrm{TeV}$, together with expected upper bounds. Solid - theoretical effective cross sections. Round markers - luminosity scaling. Dashed - luminosity scaling and pileup scaling. Magenta $-\mathcal{L}=35.9 \mathrm{fb}^{-1}$, blue $-\mathcal{L}=100 \mathrm{fb}^{-1}$, green $-\mathcal{L}=300 \mathrm{fb}^{-1}$. 


\section{B Closed-production signatures — diphoton cross sections}

The diphoton production cross sections, from a bound state resonance, with observed and future-projected upper limits at $\sqrt{s}=13 \mathrm{TeV}$ are presented in figure 16 .

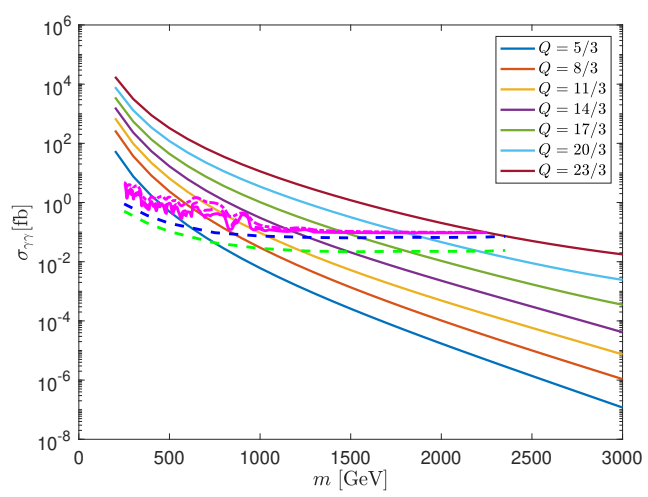

(a) Positvely-charged colored scalars.

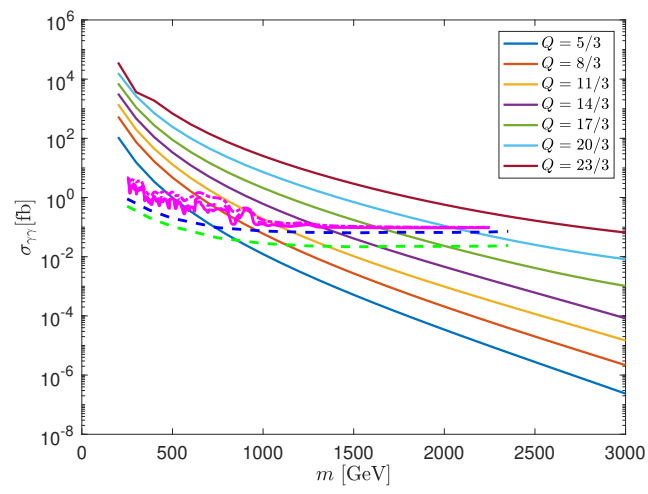

(c) Positvely-charged colored fermions.

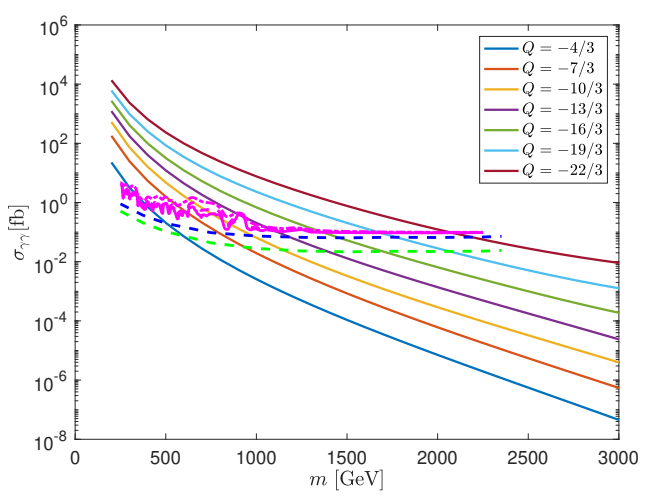

(b) Negatively-charged colored scalars.

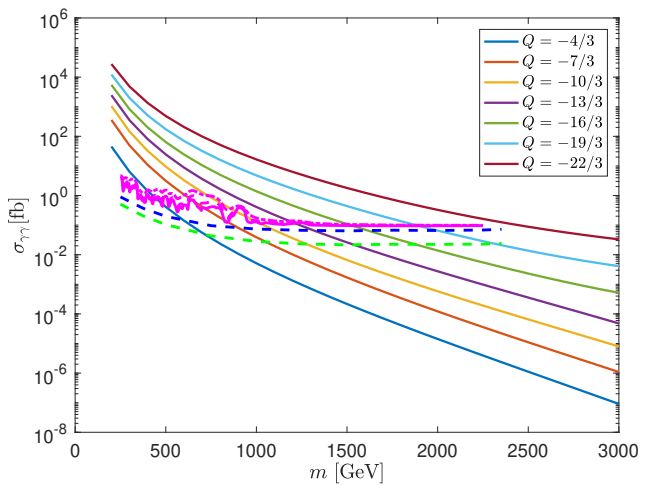

(d) Negatively-charged colored fermions.

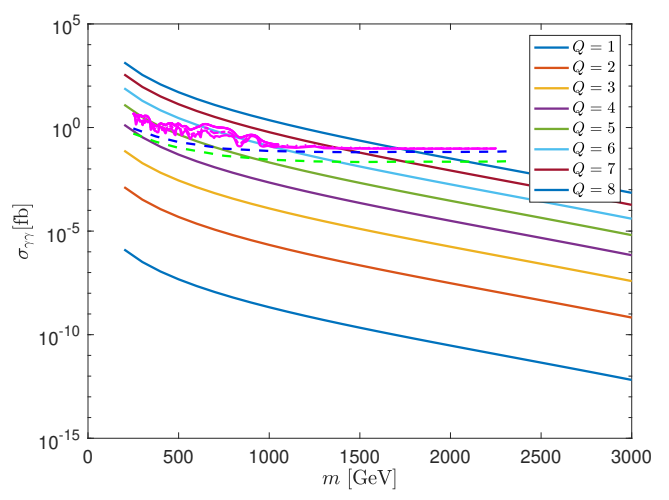

(e) Colorless fermions.

Figure 16. Diphoton resonant production cross sections, given by a bound state of mass $2 m$ at $\sqrt{s}=13 \mathrm{TeV}$. Magenta - upper-limits observed at $\mathcal{L}=35.9 \mathrm{fb}^{-1}[33]$, (solid - narrow, dashed - mid-width, dash-dotted - wide). Dashed blue — upper limits expected at $\mathcal{L}=100 \mathrm{fb}^{-1}$ [41]. Dashed green - upper-limits expected at $\mathcal{L}=300 \mathrm{fb}^{-1}[41]$. 
Open Access. This article is distributed under the terms of the Creative Commons Attribution License (CC-BY 4.0), which permits any use, distribution and reproduction in any medium, provided the original author(s) and source are credited.

\section{References}

[1] S.P. Martin, A Supersymmetry primer, hep-ph/9709356 [INSPIRE].

[2] K. Agashe and G. Servant, Warped unification, proton stability and dark matter, Phys. Rev. Lett. 93 (2004) 231805 [hep-ph/0403143] [INSPIRE].

[3] G.F. Giudice, Naturally Speaking: The Naturalness Criterion and Physics at the LHC, arXiv:0801.2562 [INSPIRE].

[4] Y. Kats, M. McCullough, G. Perez, Y. Soreq and J. Thaler, Colorful Twisted Top Partners and Partnerium at the LHC, JHEP 06 (2017) 126 [arXiv:1704.03393] [INSPIRE].

[5] Y. Kats and M.J. Strassler, Probing Colored Particles with Photons, Leptons and Jets, JHEP 11 (2012) 097 [Erratum ibid. 1607 (2016) 009] [arXiv: 1204.1119] [INSPIRE].

[6] A.C. Kraan, Interactions of heavy stable hadronizing particles, Eur. Phys. J. C 37 (2004) 91 [hep-ex/0404001] [INSPIRE].

[7] ATLAS collaboration, Search for heavy long-lived charged R-hadrons with the ATLAS detector in 3.2 $\mathrm{fb}^{-1}$ of proton-proton collision data at $\sqrt{s}=13 \mathrm{TeV}$, Phys. Lett. B 760 (2016) 647 [arXiv: 1606.05129] [INSPIRE].

[8] ATLAS collaboration, Searches for heavy long-lived charged particles with the ATLAS detector in proton-proton collisions at $\sqrt{s}=8 \mathrm{TeV}$, JHEP 01 (2015) 068 [arXiv:1411.6795] [INSPIRE].

[9] ATLAS collaboration, Searches for heavy long-lived sleptons and R-Hadrons with the ATLAS detector in pp collisions at $\sqrt{s}=7 \mathrm{TeV}$, Phys. Lett. B 720 (2013) 277 [arXiv:1211.1597] [INSPIRE].

[10] CMS collaboration, Search for long-lived charged particles in proton-proton collisions at $\sqrt{s}=13$ TeV, Phys. Rev. D 94 (2016) 112004 [arXiv:1609.08382] [InSPIRE].

[11] CMS collaboration, Searches for Long-Lived Charged Particles in pp Collisions at $\sqrt{s}=7$ and $8 \mathrm{TeV}$, JHEP 07 (2013) 122 [arXiv: 1305.0491] [INSPIRE].

[12] CMS collaboration, Search for Heavy Stable Charged Particles in pp collisions at $\sqrt{s}=7 \mathrm{TeV}$, JHEP 03 (2011) 024 [arXiv:1101.1645] [INSPIRE].

[13] ATLAS collaboration, Search for heavy long-lived multi-charged particles in pp collisions at $\sqrt{s}=8 \mathrm{TeV}$ using the ATLAS detector, Eur. Phys. J. C 75 (2015) 362 [arXiv:1504.04188] [INSPIRE].

[14] N.D. Barrie, A. Kobakhidze, S. Liang, M. Talia and L. Wu, Exotic Lepton Searches via Bound State Production at the LHC, Phys. Lett. B 781 (2018) 364 [arXiv:1710.11396] [INSPIRE].

[15] A. Manohar, P. Nason, G.P. Salam and G. Zanderighi, How bright is the proton? A precise determination of the photon parton distribution function, Phys. Rev. Lett. 117 (2016) 242002 [arXiv: 1607.04266] [INSPIRE].

[16] NNPDF collaboration, Parton distributions with QED corrections, Nucl. Phys. B 877 (2013) 290 [arXiv:1308.0598] [INSPIRE]. 
[17] Y. Kats and M.J. Strassler, Probing Colored Particles with Photons, Leptons and Jets, JHEP 11 (2012) 097 [Erratum ibid. 1607 (2016) 009] [arXiv: 1204.1119] [INSPIRE].

[18] A.V. Manohar, P. Nason, G.P. Salam and G. Zanderighi, The Photon Content of the Proton, JHEP 12 (2017) 046 [arXiv: 1708.01256] [INSPIRE].

[19] J. Alwall et al., The automated computation of tree-level and next-to-leading order differential cross sections and their matching to parton shower simulations, JHEP 07 (2014) 079 [arXiv: 1405.0301] [INSPIRE].

[20] T. Sjöstrand et al., An Introduction to PYTHIA 8.2, Comput. Phys. Commun. 191 (2015) 159 [arXiv: 1410.3012] [inSPIRE].

[21] T. Sjöstrand, S. Mrenna and P.Z. Skands, PYTHIA 6.4 Physics and Manual, JHEP 05 (2006) 026 [hep-ph/0603175] [INSPIRE].

[22] CMS collaboration, Search for multi-charged Heavy Stable Charged Particles, CMS-PAS-EXO-11-090 (2011).

[23] V. Veeraraghavan, Search for multiply charged Heavy Stable Charged Particles in data collected with the CMS detector, Ph.D. Thesis, Florida State University, Tallahassee U.S.A. (2013).

[24] M. Konecki, The RPC based trigger for the CMS experiment at the LHC, 2014 JINST 9 C07002 [INSPIRE].

[25] M. Tytgat et al., The Upgrade of the CMS RPC System during the First LHC Long Shutdown, PoS (RPC2012) 063 [arXiv: 1209.1979] [INSPIRE].

[26] V. Gori, The CMS High Level Trigger, Int. J. Mod. Phys. Conf. Ser. 31 (2014) 1460297 [arXiv: 1403.1500] [INSPIRE].

[27] Particle Data Group collaboration, Review of Particle Physics, Chin. Phys. C 40 (2016) 100001 [INSPIRE].

[28] Key Data for Ionizing-Radiation Dosimetry: Measurement Standards and Applications. Vol. 14, International Commission on Radiation Units and Measurements, Inc. (ICRU), Bethesda U.S.A. (2014). Results are taken from http://pdg.lbl.gov/2017/AtomicNuclearProperties/.

[29] R. Mackeprang, Stable Heavy Hadrons in ATLAS, Ph.D. Thesis, Bohr Institute, Copenhagen Denmark (2007).

[30] CMS collaboration, Performance of muon identification in pp collisions at $S^{0.5}=7 \mathrm{TeV}$, CMS-PAS-MUO-10-002 (2010).

[31] J. Pumplin, D.R. Stump, J. Huston, H.L. Lai, P.M. Nadolsky and W.K. Tung, New generation of parton distributions with uncertainties from global QCD analysis, JHEP 07 (2002) 012 [hep-ph/0201195] [inSPIRE].

[32] K. Blum, A. Efrati, C. Frugiuele and Y. Nir, Exotic colored scalars at the LHC, JHEP 02 (2017) 104 [arXiv: 1610.06582] [InSPIRE].

[33] CMS collaboration, Search for physics beyond the standard model in the high-mass diphoton spectrum at $13 \mathrm{TeV}$, CMS-PAS-EXO-17-017 (2018).

[34] S.P. Martin, Diphoton decays of stoponium at the Large Hadron Collider, Phys. Rev. D 77 (2008) 075002 [arXiv:0801.0237] [INSPIRE]. 
[35] J.E. Younkin and S.P. Martin, QCD corrections to stoponium production at hadron colliders, Phys. Rev. D 81 (2010) 055006 [arXiv:0912.4813] [INSPIRE].

[36] M. Beneke, J. Piclum, C. Schwinn and C. Wever, NNLL soft and Coulomb resummation for squark and gluino production at the LHC, JHEP 10 (2016) 054 [arXiv:1607.07574] [INSPIRE].

[37] Y. Kats and M.D. Schwartz, Annihilation decays of bound states at the LHC, JHEP 04 (2010) 016 [arXiv:0912.0526] [INSPIRE].

[38] D.B. Clark, E. Godat and F.I. Olness, ManeParse: A Mathematica reader for Parton Distribution Functions, Comput. Phys. Commun. 216 (2017) 126 [arXiv:1605.08012] [INSPIRE].

[39] ATLAS, CMS and The LHC Higgs Combination Group collaborations, Procedure for the LHC Higgs boson search combination in Summer 2011, CMS-NOTE-2011-005 (2011).

[40] ATLAS collaboration, Measurement of the double-differential high-mass Drell-Yan cross section in pp collisions at $\sqrt{s}=8 \mathrm{TeV}$ with the ATLAS detector, JHEP 08 (2016) 009 [arXiv: 1606.01736] [INSPIRE].

[41] E. Molinaro and N. Vignaroli, Diphoton Resonances at the LHC, Mod. Phys. Lett. A 32 (2017) 1730024 [arXiv: 1707.00926] [INSPIRE].

[42] ATLAS collaboration, Search for heavy long-lived multi-charged particles in proton-proton collisions at $\sqrt{s}=13 \mathrm{TeV}$ using the ATLAS detector, Submitted to: Phys. Rev. (2018) [arXiv: 1812.03673] [INSPIRE].

[43] CMS collaboration, Technical proposal for a MIP timing detector in the CMS experiment phase 2 upgrade, CERN-LHCC-2017-027 (2017).

[44] Y. Vibhuti, S.N.L. Sirisha and B. Sonali, Study of Stopping Power for proton in different materials - A Geant4 Based Simulation, DAE Symp. Nucl. Phys. 57 (2012) 734 [INSPIRE].

[45] CMS collaboration, RPC hit contribution to CMS muon reconstruction at LHC, Nucl. Instrum. Meth. A 718 (2013) 437 [INSPIRE].

[46] CMS collaboration, Precise Mapping of the Magnetic Field in the CMS Barrel Yoke using Cosmic Rays, 2010 JINST 5 T03021 [arXiv:0910.5530] [INSPIRE]. 